\title{
Further clues to the nature of composite LINER/H II galaxies
}

\author{
M. E. Filho ${ }^{1,2,3}$, F. Fraternali ${ }^{1,4}$, S. Markoff ${ }^{5, \star}$, N. M. Nagar ${ }^{1}$, P. D. Barthel ${ }^{1}$, L. C. Ho $^{6}$, and F. Yuan ${ }^{7}$ \\ 1 Kapteyn Astronomical Institute, PO Box 800, 9700 AV Groningen, The Netherlands \\ 2 Istituto di Radioastronomia, CNR, via P. Gobetti, 101, 40129 Bologna, Italy \\ 3 Centro de Astrofísica da Universidade do Porto, Rua das Estrelas, 4150 - 762 Porto, Portugal \\ 4 ASTRON, PO Box 2, 7990 AA Dwingeloo, The Netherlands \\ 5 Massachusetts Institute of Technology, Center for Space Research, 77 Massachusetts Av., R. NE80 - 6035, Cambridge, \\ Massachusetts 02139, USA \\ 6 The Observatories of the Carnegie Institution of Washington, 813 Santa Barbara Street, Pasadena, California 91101, USA \\ 7 Purdue University, Department of Physics, 525 Northwestern Av., West Lafayette, Indiana, 47907 - 2036, USA
}

Received 9 October 2003 / Accepted 14 January 2004

\begin{abstract}
We have analyzed new, archival and published high resolution radio and X-ray observations of a sample of composite LINER/H II galaxies known to exhibit AGN-like properties. Five of the 16 AGN candidates have milliarcsecond-scale detections and are found to display a compact, flat spectrum, high brightness temperature radio core, four of which also exhibit extended radio emission. Five of the eight AGN candidates with available high resolution X-ray observations were found to possess a hard $\mathrm{X}$-ray nuclear source, two of which have no milliarcsecond radio detection. The combined high resolution radio and X-ray data yield a 50\% detection rate of low luminosity AGN among the AGN candidates, which translates into a 12\% detection rate for the entire composite LINER/H II sample. In the sources where the AGN has been unambiguously detected, the ionizing power of the AGN is not sufficient to generate the observed emission lines, unless the hard X-rays are heavily obscured. We attempt to apply a canonical advection-dominated accretion flow (ADAF) and jet model to the sample sources in order to explain the observed radio and X-ray emission. While ADAFs may be responsible for the observed emission in submillijansky radio cores like NGC 7331, they do not appear consistent with the radio emission observed in the milliarcsecond-scale radio detected cores; the latter sources are more likely to have an energetically important contribution from a radio-emitting jet.
\end{abstract}

Key words. galaxies: active - galaxies: nuclei - radiation mechanisms: general

\section{Introduction}

The radio emission observed in nearby galaxies can be attributed to stellar and interstellar processes - non-thermal synchrotron radiation from electrons accelerated by supernova remnants (SNRs) and thermal emission from H II regions or to the presence of accreting black holes. In the former case, the radio emission coincides with the infrared and optical emission-line sources, which are themselves tracers of young stars and supernovae. The radio emission originates in diffuse, low surface brightness regions that do not extend beyond the stellar light distribution. On the other hand, radio sources produced by accreting black holes are generally characterized by compact, nuclear radio cores, sometimes associated with jets and lobes. In many instances circumnuclear starburst regions and black holes coexist spatially, making it difficult to disentangle the two components in the optical regime and also in the radio. Furthermore, at high resolution, even star-forming galaxies may show compact radio sources, associated with radio supernovae (RSN) and SNRs (e.g.

Send offprint requests to: M. E. Filho, e-mail: mfilho@ira.cnr.it

* NSF Astronomy \& Astrophysics Postdoctoral Fellow.
Weiler et al. 1986). In an active galactic nucleus (AGN), the presence of a flat radio spectrum source $\left(\alpha \lesssim 0.5 ; F_{v} \propto v^{-\alpha}\right)$ indicates synchrotron self-absorption in components whose brightness temperatures are comparable to the kinetic temperatures $\left(m c^{2} / k ; T_{B} \gtrsim 10^{5} \mathrm{~K}\right)$ of the relativistic electrons producing the synchrotron radiation. Steep spectrum emission in star-forming galaxies $(\alpha \gtrsim 0.5)$ arises from the non-thermal emission of SNRs, whereas flat spectra are consistent with freefree absorption of optically thin synchrotron radiation and/or thermal emission $\left(T_{B} \lesssim 10^{4} \mathrm{~K}\right)$ originating from the electrons in $\mathrm{H}$ II regions. So, while the spectra of thermal emitters may mimic those of partially opaque synchrotron sources, a combination of brightness temperature and radio spectral index of the strong nuclear radio components can indicate whether the emission is thermal $\left(T_{B} \lesssim 10^{4} \mathrm{~K}\right.$; Condon 1992) or non-thermal $\left(T_{B}>10^{5} \mathrm{~K}\right)$ and whether optically thin thermal $(\alpha \sim 0.1)$, self-absorbed synchrotron $(\alpha<0.5)$, or optically thin synchrotron $(\alpha \sim 0.5-0.8)$ radiation dominates.

We have therefore applied this radio technique to a sample of mildly active galaxies - composite LINER/H II galaxies - known to exhibit both starburst and AGN characteristics in their optical spectra. The motivation for studying this class 
of galaxies is to study the demographics of black hole accretion in cases suspected of weak AGN activity. This is an important issue because, if composite sources do indeed harbour a genuine active nucleus, then their considerable number $(13 \%$ of emission-line nuclei with $B_{T}<12.5 \mathrm{mag}$; Ho et al. 1997) will imply a significant contribution to the AGN population.

\section{Sample selection}

In Filho et al. $(2000,2002)$ we have presented a study of the radio characteristics of a complete sample of composite LINER/H II galaxies, i.e., galaxies that are hypothesized to be LINERs spatially contaminated by circumnuclear star-forming regions (Ho 1996). The first part of our project consisted in gathering arcsecond-scale radio information (published or new) of the 65 composite sources contained in the magnitudelimited Palomar survey (Ho et al. 1995, 1997a). Their radio properties (Filho et al. 2000, 2002) indicate composite sources come in two types: AGN-like sources with compact cores and starburst-like sources dominated by diffuse emission, co-spatial with the projected galactic disk. The complete composite source sample contained $14(\sim 25 \%)$ AGN candidates as revealed by compact, flat/inverted radio spectrum cores on arcsecond scales, with peak flux densities above $1 \mathrm{mJy}$. NGC 660 and NGC 7331 have been included in the AGN candidate sample although they have sub-mJy radio peaks as explained in Filho et al. (2002). These sixteen sources comprise the present sample of candidate AGN-driven composite LINER/H II galaxies.

\section{New and published radio observations}

Radio imaging at sub-arcsec and mas resolution of several of the sample sources have already been published. Our observations, using the Very Large Array (VLA) and the Very Large Baseline Array (VLBA), complement these observations and yield a complete picture of the occurrence of compact nuclei in the sample.

In this section we report on high resolution VLA observations of 12 (four new and eight published) and multiwavelength VLBA observations of 15 (13 new and two published) out of the 16 sample sources. Regarding the former, of the possible eight galaxies with no published sub-arcsec-scale radio information, four were chosen to fit the observational window assigned to us by the VLA. As for the latter, NGC 7331 was not observed with the VLBA because its radio core flux density at 1 .'5 resolution is well below $1 \mathrm{mJy}$ at both 1.4 and $5 \mathrm{GHz}$ (Cowan et al. 1994) and NGC 5866 and NGC 4552 because they had been previously observed and detected with the VLBA by Falcke et al. (2000) and Nagar et al. (2002), respectively. Following a description of our observations, we will combine the new and published data in a coherent analysis of the sample.

\subsection{Observations and data reduction}

VLA observations of four sources in the sample were performed on 1999 September 5 with the A-array, X-band system $(8.4 \mathrm{GHz})$. Two IFs of $50 \mathrm{MHz}$ each were combined to give a total bandwidth of $100 \mathrm{MHz}$. In its A-array configuration, the VLA yields typical resolutions of 0.25 at $8.4 \mathrm{GHz}$ (which corresponds to $12 \mathrm{pc}$ at $D=10 \mathrm{Mpc}$ ). Observations of the sample sources were interspersed with observations of nearby phase calibrators, for a total integration time of about 10 min per galaxy. The primary flux calibrator was 3C $286(1328+307)$ with adopted $8.4 \mathrm{GHz}$ flux densities of $5.1915 \mathrm{Jy}$ and $5.1702 \mathrm{Jy}$ for IF1 and IF2, respectively. The uncertainty associated with the flux calibration procedure is mainly due to the uncertainty in the absolute flux density of $3 \mathrm{C} 286$, which is conservatively set to $5 \%$. Antenna gains were found to behave well throughout the observations.

VLBA observations of 13 out of the 16 sample galaxies were obtained in five sessions - 2000 June 22, 2001 September 1, 2001 September 8, 2001 September 17 and 2001 October 6 - with the standard ten VLBA antennas. The standard observing frequency was $5 \mathrm{GHz}$, although some sources have multi-wavelength observations. The four $8 \mathrm{MHz}$ IFs yielded a total bandwidth of $32 \mathrm{MHz}$. Typical resolutions for the VLBA at $5 \mathrm{GHz}$ are 2 mas (which corresponds to $0.1 \mathrm{pc}$ at $D=10 \mathrm{Mpc}$ ). Phase referencing was performed, which includes several minute on-source scans alternated with scans of nearby phase calibrators, yielding generally 180 (in some cases 45) min total integration time on each target source.

Reduction of both the VLA and VLBA data was performed using standard NRAO AIPS (version 15Oct99) image processing routines. After initial deleting of bad data points and calibration, the data were "cleaned". The AIPS "cleaning" task IMAGR was employed to Fourier transform the data and remove sidelobes from the maps in an interactive and iterative mode. Some sources were observed in multiple VLBA runs separated by several days. For these sources, maps were made for each run separately, since combining the data did not result in a better map. Full resolution images, having a 0.'25 synthesized beam, were obtained for the VLA data and 2 mas synthesized beam at $5 \mathrm{GHz}$ for the VLBA data. The image noise level reached the theoretical level of $\sim 0.07 \mathrm{mJy} / \mathrm{beam}$ (uniform weighting; Perley et al. 1989) to within a factor of two for the VLA data and $\sim 0.05 \mathrm{mJy} /$ beam for the VLBA data. Phase selfcalibration was employed on the strongest sources, leading to some reduction in the image noise level. The $8.4 \mathrm{GHz}$ VLA calibrated data provided by J. Ulvestad for NGC 660 have been re-analysed, yielding slightly different radio parameters than those presented in Filho et al. (2002).

The image noise levels were measured with the AIPS task IMSTAT in a source-free region. Using the AIPS task IMFIT, the brightness peaks of the radio sources were fitted with single bi-dimensional Gaussians.

\subsection{Results of the radio observations}

Four of four VLA and three of 13 newly observed VLBA sample sources were detected. Table 1 lists image parameters for the new data, where we have also included relevant published radio data from Nagar et al. (2000, 2002), Cowan et al. (1994; NGC 7331) and Falcke et al. (2000; NGC 5866). We refer to Filho et al. $(2000,2002)$ for an 
Table 1. Map parameters (published or new) of the sample sources. Column 1: source name. Column 2: observing frequency. Column 3: integration time. Column 4: rms noise level of the image. Column 5: restoring beam or resolution. Column 6: position angle of the beam. Column 7: peak radio flux density. Column 8: integrated radio flux density. Columns 9 and 10: radio position. Column 11: deconvolved source size. Column 12: position angle of the source. Value in parenthesis is an uncertain value. Column 13: note. Letter is the observing epoch and number is the reference (published or new) from which the radio data were taken.

\begin{tabular}{|c|c|c|c|c|c|c|c|c|c|c|c|c|}
\hline $\begin{array}{l}\text { Galaxy } \\
\text { (1) }\end{array}$ & $\begin{array}{c}v \\
(\mathrm{GHz}) \\
(2)\end{array}$ & $\begin{array}{c}\tau \\
(\min ) \\
(3)\end{array}$ & $\begin{array}{c}\mathrm{rms} \\
\left(\frac{\mathrm{mJy}}{\text { beam }}\right) \\
(4)\end{array}$ & $\begin{array}{c}\text { Beamsize } \\
\left(\operatorname{arcsec}^{2}\right) \\
(5)\end{array}$ & $\begin{array}{c}\text { PA } \\
(\mathrm{deg}) \\
(6)\end{array}$ & $\begin{array}{c}F_{\text {peak }} \\
\left(\frac{\mathrm{mJy}}{\text { beam }}\right) \\
(7)\end{array}$ & $\begin{array}{c}F_{\text {int }} \\
(\mathrm{mJy}) \\
(8)\end{array}$ & $\begin{array}{c}\mathrm{RA}(\mathrm{J} 2000) \\
\left(\mathrm{h} \mathrm{m} \mathrm{s}^{\circ}\right) \\
(9)\end{array}$ & $\begin{array}{c}\operatorname{Dec}(\mathrm{J} 2000) \\
\left({ }^{\circ}{ }^{\prime \prime \prime}\right) \\
(10)\end{array}$ & $\begin{array}{c}\text { Size } \\
\left(\operatorname{arcsec}^{2}\right) \\
(11)\end{array}$ & $\begin{array}{c}\text { PA } \\
(\mathrm{deg}) \\
(12)\end{array}$ & $\begin{array}{l}\text { Note } \\
(13)\end{array}$ \\
\hline \multicolumn{13}{|l|}{ VLA Obs. } \\
\hline \multirow[t]{2}{*}{ NGC 660} & 8.4 & 270 & 0.01 & $0.21 \times 0.21$ & -39.90 & 0.5 & 2.3 & 014302.32 & +133844.9 & $0.46 \times 0.36$ & 174.30 & $1, \mathrm{a}$ \\
\hline & 15 & 16 & 0.20 & 0.15 & $\ldots$ & $<0.9$ & $<0.9$ & $\ldots$ & $\ldots$ & $\ldots$ & $\ldots$ & 2 \\
\hline NGC 1055 & 15 & 16 & 0.20 & 0.15 & $\ldots$ & $<1.8$ & $<1.8$ & $\ldots$ & $\ldots$ & $\ldots$ & $\ldots$ & 2 \\
\hline NGC 3627 & 15 & 16 & 0.20 & 0.15 & $\ldots$ & 1.1 & 2.9 & 112015.00 & +125929.6 & $\ldots$ & $\ldots$ & 2 \\
\hline NGC 4216 & 15 & 16 & 0.20 & 0.15 & $\ldots$ & 1.2 & 1.3 & 121554.37 & +130858.0 & $\ldots$ & $\ldots$ & 2 \\
\hline NGC 4419 & 15 & 16 & 0.20 & 0.15 & $\ldots$ & 2.7 & 3.6 & 122656.45 & +150250.7 & $\ldots$ & $\ldots$ & 2 \\
\hline NGC 4527 & 15 & 16 & 0.20 & 0.15 & $\ldots$ & $<1.1$ & $<1.1$ & $\ldots$ & $\ldots$ & $\ldots$ & $\ldots$ & 3 \\
\hline \multirow[t]{2}{*}{ NGC 4552} & 8.4 & 10 & 0.15 & $0.25 \times 0.21$ & -27.79 & 102.1 & 102.2 & 123539.80 & +123322.7 & $\ldots$ & $\ldots$ & $4, b$ \\
\hline & 15 & 16 & 0.20 & 0.15 & & 58.1 & 58.6 & 123539.80 & +123322.7 & $\ldots$ & $\ldots$ & 2 \\
\hline NGC 5354 & 8.4 & 10 & 0.12 & $0.26 \times 0.24$ & 73.41 & 9.7 & 10.1 & 135326.70 & +401810.0 & $\ldots$ & 15.73 & $4, b$ \\
\hline NGC 5838 & 8.4 & 10 & 0.07 & $0.31 \times 0.23$ & 36.77 & 1.6 & 1.6 & 150526.24 & +020557.4 & $\ldots$ & $\ldots$ & $4, b$ \\
\hline NGC 5846 & 8.4 & 10 & 0.10 & $0.30 \times 0.23$ & 35.29 & 6.3 & 6.4 & 150629.28 & +013620.4 & $\ldots$ & $\ldots$ & $4, b$ \\
\hline NGC 5866 & 15 & 16 & 0.20 & 0.15 & $\ldots$ & 7.1 & 7.5 & 150629.49 & +554547.5 & $\ldots$ & $\ldots$ & 3 \\
\hline \multirow[t]{3}{*}{ NGC 7331} & 1.5 & $\ldots$ & 0.03 & $1.8 \times 1.4$ & $\ldots$ & 0.2 & 0.23 & 223704.06 & +34 2456.9 & $\ldots$ & $\ldots$ & 5 \\
\hline & 5 & $\ldots$ & 0.02 & $1.8 \times 1.4$ & $\ldots$ & 0.1 & 0.12 & 223704.06 & +342456.9 & $\ldots$ & $\ldots$ & 5 \\
\hline & 15 & 16 & 0.20 & 0.15 & $\ldots$ & $<1.1$ & $<1.1$ & $\ldots$ & $\ldots$ & $\ldots$ & $\ldots$ & 2 \\
\hline $\begin{array}{l}\text { Galaxy } \\
\text { (1) }\end{array}$ & $\begin{array}{c}v \\
(\mathrm{GHz}) \\
(2)\end{array}$ & $\begin{array}{c}\tau \\
(\min ) \\
(3)\end{array}$ & $\begin{array}{c}\mathrm{rms} \\
\left(\frac{\mathrm{mJy}}{\mathrm{beam}}\right) \\
(4)\end{array}$ & $\begin{array}{c}\text { Beamsize } \\
\left(\mathrm{mas}^{2}\right) \\
(5)\end{array}$ & $\begin{array}{c}\text { PA } \\
\text { (deg) } \\
(6)\end{array}$ & $\begin{array}{c}F_{\text {peak }} \\
\left(\frac{\text { mJy }}{\text { beam }}\right) \\
(7)\end{array}$ & $\begin{array}{c}F_{\text {int }} \\
(\mathrm{mJy}) \\
(8)\end{array}$ & $\begin{array}{c}\text { RA(J2000) } \\
\left(\begin{array}{c}\mathrm{h} \mathrm{m} \mathrm{s}) \\
(9)\end{array}\right.\end{array}$ & $\begin{array}{c}\operatorname{Dec}(\mathrm{J} 2000) \\
\left({ }^{\prime}, " \prime\right) \\
(10)\end{array}$ & $\begin{array}{c}\begin{array}{c}\text { Size } \\
\left(\mathrm{mas}^{2}\right)\end{array} \\
(11)\end{array}$ & $\begin{array}{c}\text { PA } \\
(\operatorname{deg}) \\
(12)\end{array}$ & $\begin{array}{c}\text { Note } \\
(13)\end{array}$ \\
\hline \multicolumn{13}{|l|}{ VLBA Obs. } \\
\hline NGC 410 & 5 & 180 & 0.08 & $3.6 \times 1.9$ & -7.91 & $<0.4$ & $<0.4$ & $\ldots$ & & $\ldots$ & $\ldots$ & $4, c$ \\
\hline NGC 524 & 5 & 180 & 0.08 & $4.5 \times 2.0$ & -9.89 & 0.9 & 1.5 & 012447.746 & +093220.14 & $2.8 \times 1.8$ & 168.02 & $4, c$ \\
\hline NGC 660 & 5 & 180 & 0.09 & $4.4 \times 1.9$ & -8.73 & $<0.5$ & $<0.5$ & $\ldots$ & $\ldots$ & $\ldots$ & $\ldots$ & $4, d$ \\
\hline NGC 1055 & 5 & 180 & 0.08 & $5.0 \times 1.9$ & -16.32 & $<0.4$ & $<0.4$ & $\ldots$ & $\ldots$ & $\ldots$ & $\ldots$ & $4, c$ \\
\hline NGC 1161 & 5 & 180 & 0.08 & $3.2 \times 1.8$ & -10.47 & $<0.5$ & $<0.5$ & $\ldots$ & $\ldots$ & $\ldots$ & $\ldots$ & $4, \mathrm{~d}$ \\
\hline NGC 3245 & 5 & 172 & 0.09 & $3.8 \times 1.7$ & -4.70 & $<0.5$ & $<0.5$ & $\ldots$ & $\ldots$ & $\ldots$ & $\ldots$ & $4, \mathrm{e}$ \\
\hline NGC 3627 & 5 & 96 & 0.09 & $3.6 \times 1.4$ & -8.62 & $<0.3$ & $<0.3$ & $\ldots$ & $\ldots$ & $\ldots$ & $\ldots$ & $4, \mathrm{f}$ \\
\hline NGC 4216 & 5 & 168 & 0.08 & $4.5 \times 1.8$ & -9.15 & $<0.4$ & $<0.4$ & $\ldots$ & $\ldots$ & $\ldots$ & $\ldots$ & $4, \mathrm{~g}$ \\
\hline NGC 4419 & 5 & 168 & 0.08 & $4.4 \times 1.8$ & -8.87 & $<0.4$ & $<0.4$ & $\ldots$ & $\ldots$ & $\ldots$ & $\ldots$ & $4, \mathrm{~g}$ \\
\hline NGC 4527 & 5 & 156 & 0.09 & $4.6 \times 1.6$ & -12.64 & $<0.5$ & $<0.5$ & $\ldots$ & $\ldots$ & $\ldots$ & $\ldots$ & $4, \mathrm{e}$ \\
\hline NGC 4552 & 5 & 52 & 0.20 & $2.9 \times 1.2$ & 2.40 & 99.5 & 99.5 & 123539.807 & +123322.83 & & & 2 \\
\hline \multirow[t]{4}{*}{ NGC 5354} & 2.3 & 40 & 0.17 & $7.6 \times 3.8$ & -3.44 & 4.5 & 8.7 & 135326.713 & +401809.93 & $5.7 \times 3.2$ & 102.02 & $4, \mathrm{~g}$ \\
\hline & 5 & 105 & 0.11 & $2.3 \times 1.6$ & 6.74 & 2.4 & 6.6 & 135326.712 & +401809.94 & $3.9 \times 0.7$ & 90.95 & $4, \mathrm{f}$ \\
\hline & 5 & 48 & 0.14 & $3.5 \times 1.7$ & -8.05 & 4.5 & 8.6 & 135326.712 & +401809.93 & $2.5 \times 1.5$ & 103.11 & $4, \mathrm{~g}$ \\
\hline & 15 & 36 & 0.25 & $1.2 \times 0.6$ & -7.43 & $<1.3$ & $<1.3$ & $\ldots$ & $\ldots$ & $\ldots$ & $\ldots$ & $4, \mathrm{~g}$ \\
\hline NGC 5838 & 5 & 96 & 0.11 & $3.4 \times 1.4$ & -1.26 & $<0.3$ & $<0.3$ & $\ldots$ & $\ldots$ & $\ldots$ & $\ldots$ & $4, \mathrm{f}$ \\
\hline NGC 5846A & 2.3 & 48 & 0.16 & $9.2 \times 3.7$ & -0.51 & 0.8 & 2.8 & 150629.292 & +013620.35 & $9.0 \times 7.8$ & 173.92 & $4, \mathrm{e}$ \\
\hline NGC 5846A & 5 & 112 & 0.05 & $3.4 \times 1.4$ & -0.71 & 0.6 & 1.1 & 150629.292 & +013620.34 & $2.8 \times 1.3$ & 7.85 & $4, \mathrm{f}$ \\
\hline NGC 5846A & 5 & 48 & 0.12 & $4.7 \times 1.8$ & -6.65 & 1.2 & 1.5 & 150629.292 & +013620.34 & $2.3 \times 2.3$ & $\ldots$ & $4, \mathrm{e}$ \\
\hline NGC 5846B & 5 & 112 & 0.05 & $3.4 \times 1.4$ & -0.71 & 0.3 & 0.6 & 150629.292 & +013620.44 & $4.5 \times 1.2$ & 175.27 & $4, \mathrm{f}$ \\
\hline NGC 5846C & 5 & 112 & 0.05 & $3.4 \times 1.4$ & -0.71 & 0.3 & 0.7 & 150629.292 & +013620.45 & $5.2 \times 1.7$ & 147.98 & $4, \mathrm{f}$ \\
\hline NGC 5846D & 15 & 48 & 0.16 & $1.7 \times 0.7$ & -9.44 & 1.4 & 1.9 & 150629.292 & +013620.40 & $1.3 \times 0.2$ & 158.17 & $3, \mathrm{e}$ \\
\hline NGC 5866 & 5 & 45 & 0.20 & $\ldots$ & $\ldots$ & 7.0 & 8.4 & 150629.499 & +554547.57 & $\ldots$ & $(11)$ & 6 \\
\hline
\end{tabular}

REFERENCES - (1) Filho et al. (2002) from calibrated dated supplied by J. Ulvestad; (2) Nagar et al. (2002); (3) Nagar et al. (2000); (4) this paper; (5) Cowan et al. (1994); (6) Falcke et al. (2000).

Observations - (a) 1995 July 13; (b) 1999 Sep. 05; (c) 2001 Sep. 01; (d) 2001 Sep. 17; (e) 2001 Oct. 06; (f) 2000 June 22; (g) 2001 Sep. 08. 


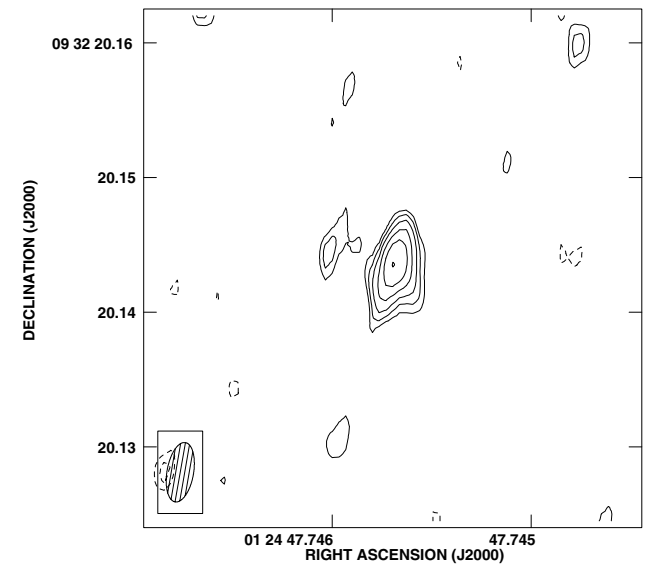

(a)

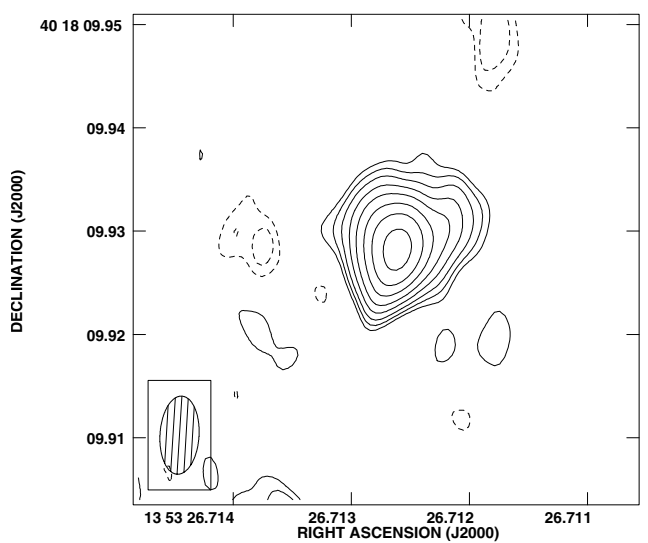

(b)

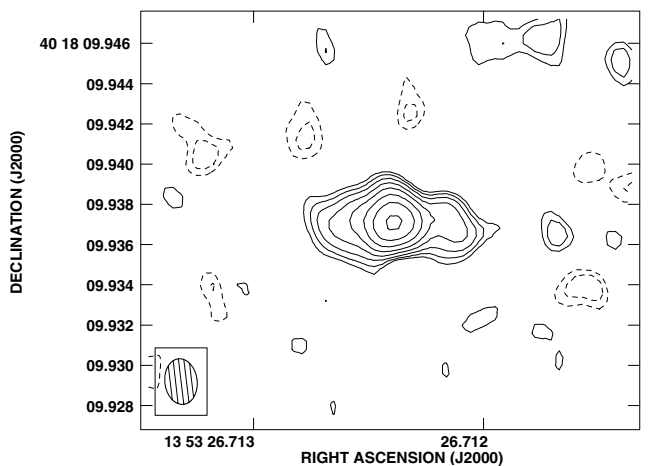

(c)

Fig. 1. Radio emission contours with the following multiples of the rms noise (Table 1): $(-3,-2.1,2.1,3,4.2,6,8.4,12,16.8,24)$. The size of the restoring beam in milliarcseconds, observing epoch, integration time in minutes, and frequency in $\mathrm{GHz}$ are given in parentheses after each object name (see Table 1). a) NGC 524 (4.5×2.0; 2001 Sep. 01; 180; 5); b) NGC 5354 (7.6×3.8; 2001 Sep. 17; 40; 2.3); c) NGC 5354 (2.3×1.6; 200 June $22 ; 105 ; 5)$.

extensive discussion on the radio properties of the sample sources. Undetected sources have been given $5 \sigma$ upper limits for the VLA and VLBA data. We estimate the radio peak positions to be accurate to within $\lesssim 0$.' 2 for the VLA and $\lesssim 1$ mas for the VLBA data, due to the good phase solutions and accurate positions of the phase calibrators. Sources are considered unresolved if their deconvolved source sizes are less than half the beamwidth in any component.

Figures 1 and 2 show the highest signal-to-noise radio maps of the newly detected VLBA sources. The contour levels are the following multiples of the rms noise (Table 1) in the map: -3 , $-2.1,2.1,3,4.2,6,8.4,12,16.8,24$.

\subsubsection{Radio core properties}

In Table 2 we list the radio properties of the detected mas-scale sample sources (from new or published data). Unless otherwise mentioned, we have used the longest integration time VLBA $5 \mathrm{GHz}$ radio flux densities to calculate the spectral indices and brightness temperatures. Radio spectral indices should be taken with caution, due to non-simultaneity and mismatch in resolution. For the multiple radio components in NGC 5846, we have given $5 \sigma$ limits to any $5 \mathrm{GHz}$ emission in order to obtain upper limits for the spectral indices. We calculate the brightness temperature as:

$T_{B}=7.8 \times 10^{6}\left(\frac{F_{v}}{\mathrm{mJy}}\right)\left(\frac{\theta}{2.5 \mathrm{mas}}\right)^{-2}\left(\frac{v}{5 \mathrm{GHz}}\right)^{-2}$

where $F_{v}$ is the peak flux density at $5 \mathrm{GHz}$ in the VLBA maps (Table 1) and $\theta$ is the FWHM of the Gaussian beam, taken to be typically 2.5 mas at $5 \mathrm{GHz}$. For unresolved sources, the quoted brightness temperatures should be considered lower limits.

In a distance-limited sample of low luminosity Seyferts, LINERs and composite LINER/H II galaxies imaged with the VLA (150 mas resolution), 50\% of the sources showed flat spectrum radio cores (Nagar et al. 2002, 2000). Subsequent VLBA $5 \mathrm{GHz}$ observations of a flux-limited subsample (16 sources with flux densities $>3 \mathrm{mJy}$ ) yielded a $100 \%$ detection rate of low luminosity AGN (LLAGN; Nagar et al. 2002; see also Falcke et al. 2000). In comparison, the present sample of AGN candidates yielded a VLA radio core detection rate of $83 \%$ (ten of 12 sources with VLA data, Table 1). Follow-up VLBA observations (including NGC 4552; Nagar et al. 2002 and NGC 5866; Falcke et al. 2000) have revealed that $33 \%$ (five of 15) of the sources have mas-scale radio cores. These results 


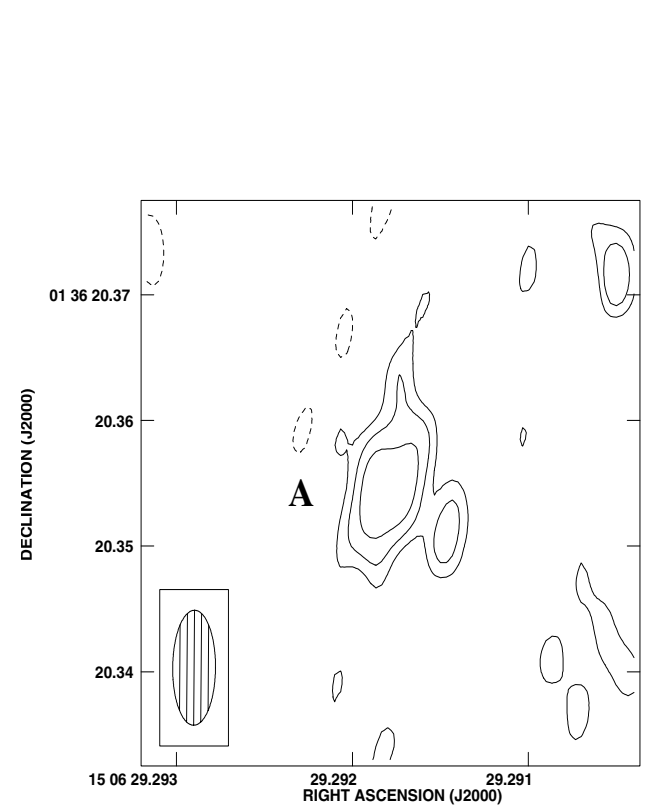

(a)

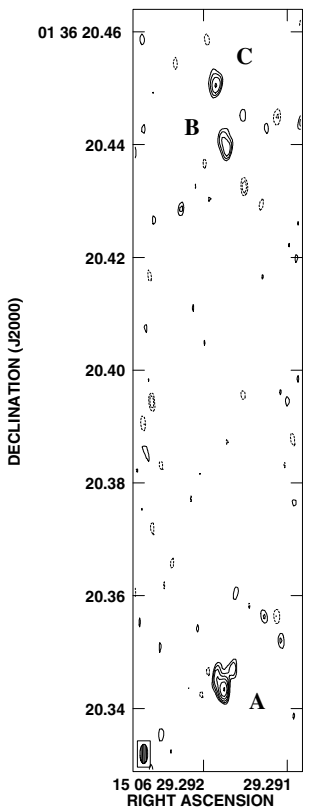

(b)

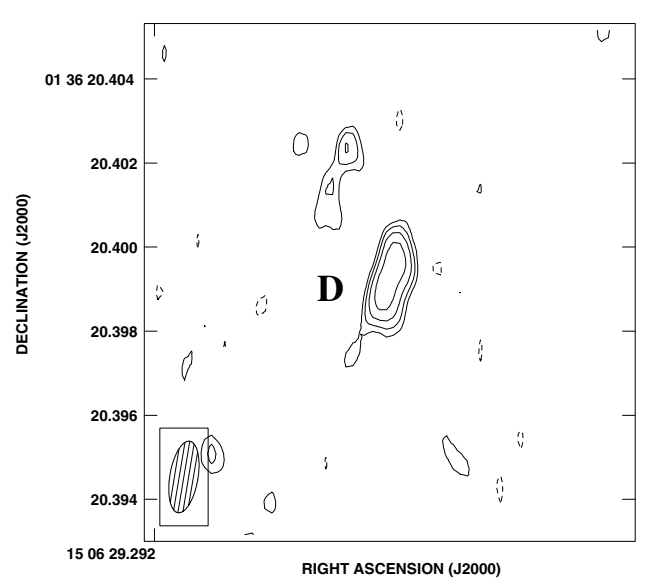

(c)

Fig. 2. Same as Fig. 1. a) NGC $5846(9.2 \times 3.7 ; 2001$ Oct. 06; 48; 2.3); b) NGC $5846(3.1 \times 1.4 ; 2000$ June 22; 112; 5); c) NGC 5846 (1.7×0.7; 2001 Oct. 06; 48; 15).

translate to an overall radio detection rate of $8 \%$ (five of 65 ) or $12 \%$ if we include NGC 660 and NGC 7331 (see Sect. 5), among the entire composite LINER/H II sample. The mas-scale non-detections were sources with VLA flux densities $<3 \mathrm{mJy}$; thus, our results are consistent with those of Falcke et al. (2000) and Nagar et al. (2002). Moreover, as has been pointed out in these papers and discussed also in Filho et al. (2002), scaling arguments suggest that the radio non-detections could well be lower power versions of the radio detections and thus harder to detect. The true AGN fraction in composite LINER/H II galaxies is likely to be higher.

Of the four VLA observed sample sources, only NGC 660 was slightly resolved at VLA A-array resolution. The present new and published VLBA data show unresolved cores in NGC 524 (Fig. 1a), NGC 4552 (Nagar et al. 2002) and NGC 5354 (Figs. 1b,c). Some sources also show evidence of radio variability, as seems the case for NGC 4552 (see discussion in Filho et al. 2000). Furthermore, all VLBA detected sample sources (including NGC 4552; Nagar et al. 2002 and NGC 5866; Falcke et al. 2000) show flat spectrum radio cores, although the inverted spectrum in NGC 524 is most probably an effect of resolution mismatch. The origin of the mas-scale radio emission is unlikely to be free-free emission. The detected radio cores show brightness temperatures several orders of magnitude above the thermal limit ( $10^{4} \mathrm{~K}$; Condon 1992). Moreover, as argued in Falcke et al. (2001), a thermal origin for the radio emission would imply much higher X-ray fluxes than those observed (see Sect. 4). Therefore, the combination of brightness temperatures in excess of $10^{7} \mathrm{~K}$ and spectral indices $\alpha \lesssim 0.5$ shows that the radio emission can be identified with (multi-component) non-thermal, self-absorbed synchrotron emission related to an accreting black hole. 
Table 2. Nuclear radio properties (published or new). Column 1: source name. Column 2: high resolution radio flux density. Column 3: observing frequencies. Column 4: radio spectral index. Column 5: radio variability. Column 6: peak brightness temperature. Column 7: note.

\begin{tabular}{|c|c|c|c|c|c|c|}
\hline $\begin{array}{l}\text { Galaxy } \\
\text { (1) }\end{array}$ & $\begin{array}{c}F_{\text {radio }} \\
(\mathrm{mJy}) \\
(2)\end{array}$ & $\begin{array}{c}v \\
(\mathrm{GHz}) \\
(3)\end{array}$ & $\begin{array}{c}\alpha \\
(4)\end{array}$ & $\begin{array}{l}\text { var. } \\
\text { (5) }\end{array}$ & $\begin{array}{l}T_{B} \\
(\mathrm{~K}) \\
(6)\end{array}$ & $\begin{array}{c}\text { Note } \\
\text { (7) }\end{array}$ \\
\hline NGC 524 & $1.95 / 1.5$ & $8.4 / 5$ & $>-0.5$ & $\ldots$ & $7.0 \times 10^{6}$ & $\mathrm{a}$ \\
\hline NGC 4552 & $102.1 / 99.5$ & $8.4 / 5$ & $\sim 0.0$ & yes & $7.8 \times 10^{8}$ & $b, c$ \\
\hline NGC 5354 & $8.6 / 8.7$ & $5 / 2.3$ & 0.0 & yes? & $1.9 \times 10^{7}$ & $\ldots$ \\
\hline NGC 5846A & $1.5 / 2.8$ & $5 / 2.3$ & 0.8 & yes? & $4.7 \times 10^{6}$ & $\ldots$ \\
\hline NGC 5846B & $0.6 /<0.8$ & $5 / 2.3$ & $>0.4$ & $\ldots$ & $2.3 \times 10^{6}$ & $\ldots$ \\
\hline NGC 5846C & $0.7 /<0.8$ & $5 / 2.3$ & $>0.2$ & $\ldots$ & $2.3 \times 10^{6}$ & $\ldots$ \\
\hline NGC 5846D & $1.9 /<0.3$ & $15 / 5$ & $>-1.8$ & yes? & $7.6 \times 10^{6}$ & $\mathrm{~d}$ \\
\hline NGC 5866 & $7.5 / 8.4$ & $15 / 5$ & 0.1 & $\ldots$ & $1.0 \times 10^{8}$ & $\mathrm{e}$ \\
\hline NGC 7331 & $0.121 / 0.234$ & $5 / 1.5$ & 0.5 & $\ldots$ & $\ldots$ & $\mathrm{f}$ \\
\hline
\end{tabular}

NoTES - (a) spectral index calculated using the $8.4 \mathrm{GHz}, 2$ '. 5 resolution peak flux density (Filho et al. 2002) and VLBA 5 GHz integrated flux density (this paper); (b) spectral index calculated using the $8.4 \mathrm{GHz}, 2$ '.5 resolution peak flux density (Filho et al. 2002) and VLBA 5 GHz integrated flux density (Nagar et al. 2002); (c) brightness temperature calculated using the VLBA $5 \mathrm{GHz}$ peak flux density (Nagar et al. 2002); (d) brightness temperature calculated using the VLBA $15 \mathrm{GHz}$ peak flux density (this paper); (e) spectral index calculated using the VLA, $15 \mathrm{GHz}, 0.15^{\prime \prime}$ resolution (Nagar et al. 2002) and VLBA $5 \mathrm{GHz}$ integrated flux density (Falcke et al. 2000); (f) spectral index calculated using the VLA, 1.5 and $5 \mathrm{GHz}, 2^{\prime \prime}$ resolution integrated flux density (Cowan et al. 1994).

On the other hand, the nature of the radio components associated with NGC5846 is more complex. Our 2.3, 5, and $15 \mathrm{GHz}$ VLBA maps of NGC 5846 show a multiple radio source roughly aligned in the North-South direction (Figs. 2a,b,c) - components NGC 5846A, NGC 5846B, NGC 5846C and NGC 5846D. Apparently we have resolved the single core observed with the VLA A-array ( $0 . ' 25$ resolution) and VLA C-array (2'.5 resolution; Filho et al. 2002). At $5 \mathrm{GHz}, 11$ mas separate the Southernmost (component A) from the Northern ones (components B and C). Component A appears in both the $2.3 \mathrm{GHz}$ and $5 \mathrm{GHz}$ maps (Figs. 2a,b), remaining unresolved at the latter frequency. However, it is not detected at $15 \mathrm{GHz}$ (Fig. 2c), showing the source to be steep spectrum and/or variable. The steepness of the spectrum and high brightness temperature (Table 2) make it a candidate RSN (e.g. Tarchi et al. 2000). However, the compactness of the source, associated with the high brightness temperature and given its linear radio structure, suggests that component $\mathrm{A}$ is the variable radio core of NGC 5846 .

\subsubsection{Extended radio nuclear components}

There are three (NGC 5354, NGC 5846 and also NGC 4552; Nagar et al. 2002), perhaps four (see discussion on NGC 5866 in Falcke et al. 2000) sources in the sample which show extended structure on mas-scales.

Although unresolved on arcsec-scales (Filho et al. 2000; Nagar et al. 2000), and in our VLBA maps, a recent high signalto-noise VLBA image of NGC 4552 (Nagar et al. 2002) revealed a core plus two-sided emission in the East and West direction.
Our 0.'2 resolution VLA map of NGC 5354 shows an unresolved core, whereas our longer integration VLBA map of this source shows about $4 \mathrm{mJy}$ in symmetric double-sided emission to the East and West (Fig. 1c), suggesting the presence of jets cradling an AGN core. This extended emission is not seen, however, on our other VLBA shorter exposure maps, most probably due to low signal-to-noise.

Our first epoch $5 \mathrm{GHz}$ VLBA map shows a triple source which we have designated as components NGC 5846A, B, and $\mathrm{C}$. Furthermore, at $15 \mathrm{GHz}$ we detect another component (NGC 5846D; Fig. 2c), about $\lesssim 6$ mas from the $5 \mathrm{GHz}$ radio position of NGC 5846A and which cannot be identified with any of the components in the $5 \mathrm{GHz}$ map. NGC 5846A has been discussed in the previous subsection, but the nature of the latter three components is more difficult to constrain. Components B, C and D appear only at one frequency each, and most probably have an inverted (NGC 5846D) or steep spectrum (NGC 5846B and NGC 5846C), suggestive of optically thin synchrotron emission. The apparent inverted nature of the spectrum of NGC 5846D could also be due to radio variability. All three components also appear to have a somewhat amorphous, resolved morphology and high brightness temperatures typical for non-thermal sources (Table 2). Furthermore, they are more or less aligned with component A in the North-South direction. One scenario is that these components are compact SNRs near the center of the galaxy (e.g. Tarchi et al. 2000) or they may be emission associated with a radio jet.

\section{Archival and published X-ray observations}

For most of our sample sources, published X-ray data are available - they mainly refer to low resolution observations not 
suited to our purposes. Therefore, in this section we present the results of the analysis of archival and/or published high resolution X-ray Chandra data for the sample sources.

\subsection{Observations and data reduction}

Public Chandra ACIS-S archival data are available for seven of the sample sources, three of which have already been published (NGC 1055 and NGC 3627; Ho et al. 2001 and NGC 5846; Trinchieri \& Goudfrooij 2002). NGC 5866 Chandra data have been published (Terashima \& Wilson 2003) but are not as yet publicly available.

For reasons of self-consistency and homogeneity, all publicly available Chandra data were reduced and analyzed using the CIAO (version 2.2) and XSPEC (version 11.2) packages. We removed high background time by excluding events exceeding $\pm 3 \sigma$ of the mean image count rate. The energy range was restricted to the $0.4-10 \mathrm{keV}$ band. We searched for $\mathrm{X}$-ray nuclei coincident with the radio core positions (Filho et al. 2000, 2002; Nagar et al. 2000, 2002; Sect. 3) to within the positional accuracy of Chandra $\left(\sim 1^{\prime \prime}\right)$. When found, spectra were extracted from a circular region with a diameter of $2.5^{\prime \prime}$ ( $\sim 5$ pixels on the ACIS image) in order to spatially match both the VLA C-array radio maps (Filho et al. 2000, 2002) and the regions from where the $\mathrm{H} \alpha$ luminosities were obtained (Ho et al. 1997). The background was extracted from an annulus around the source with an inner diameter of $5^{\prime \prime}$.

Possible difficulties in pinpointing a hard X-ray nucleus can arise from the presence of ultraluminous X-ray sources (ULXs). Such confusion is minimized by the high resolution of Chandra. However, this possibility can not be totally excluded for galaxies like NGC 5846 (see Sect. 4.2), where several X-ray sources are detected in the nuclear region. With the possible exception of NGC 4552, pile-up effects should not be a problem, given the relatively low count rates. Even in the case of NGC 4552, a pile-up correction with a subsequent factor 2 difference in flux would not alter the following results.

For three of the X-ray sources (NGC 4552, NGC 5846, NGC 7331), the longer exposure times $(\tau \simeq 30 \mathrm{ks})$, allowed a spectral analysis. We considered a power law model plus, for NGC 4552, a thermal plasma component (Raymond \& Smith 1977). The column densities have been fixed to the Galactic values (Dickey \& Lockman 1990). The fitted photon indices (1.5-2.3) are consistent with values obtained for LLAGN (Terashima \& Wilson 2003). For sources observed with shorter exposure times, the count rates were converted to X-ray fluxes assuming a simple power-law spectrum with a photon index of 1.7 and Galactic absorption. Upper limits for nondetections were calculated after running a source detection procedure (wavdetect) on the full resolution Chandra image, with a threshold of $10^{-6}(4.7 \sigma$ level). Assuming a power-law $(\Gamma=1.7)$ and Galactic absorption, the upper limits were calculated to be equal to the flux of the weakest detected source on the image. Our results are consistent with those obtained by Ho et al. (2001) for NGC 1055 and NGC 3627 and Trinchieri \& Goudfrooij (2002) for NGC 5846 (see Sect. 4.2).

\subsection{Results of the X-ray observations}

The X-ray parameters as given by Chandra archival or published data are specified in Table 3.

Five of eight (63\%) sample sources with available Chandra data were detected as having hard X-ray nuclear cores. Given the hypothetical weakness of the hard X-ray core, the three non-detections are most likely related to the low integration time compared to the observations of the other sources; these have $\tau=1-2 \mathrm{ks}$ (Table 3 ).

Below we give a brief description of the X-ray properties of the AGN candidates.

$N G C 660$ The soft X-ray region is about 0.5 in extent in the Northeast-Southwest direction, about the size and orientation of the radio emitting region (see Filho et al. 2002). The hard $\mathrm{X}$-ray nuclear region, spatially coincident with the radio core (Filho et al. 2002), comprises only about 10 counts, such that no spectral fit was possible.

NGC 1055 No obvious X-ray source - hard or soft - was found on the Chandra image (see also Ho et al. 2001). A source detection procedure (wavdetect) was run in order to obtain an upper limit to the X-ray emission.

NGC 3245 There is a hard nuclear X-ray source coincident with the optical nucleus. The soft X-ray emission is resolved and extended $\left(\sim 6^{\prime \prime}\right)$ in the North-South direction.

$N G C 3627$ The soft X-ray emission extends over about $2^{\prime}$ in a Northwest-Southeast direction, similar to the extent and orientation of the triple-radio source (Filho et al. 2000). No obvious nuclear X-ray source - hard or soft - was found on the Chandra image (see also Ho et al. 2001). Although the source detection procedure (wavdetect) found a source positionally coincident with the VLA core (Filho et al. 2000; Nagar et al. $2000,2002)$, it is the weakest source on the image and we prefer to consider it an upper limit (see also Ho et al. 2001).

$N G C 4552$ The soft X-ray emission extends almost to the limits of the optical galaxy $\left(\sim 2^{\prime}\right)$, exhibiting a somewhat hourglass structure. We find a strong hard X-ray nuclear source, coincident with the radio core position (Nagar et al. 2002; Filho et al. 2000). The spectrum of the nuclear source (1200 net counts) yields a satisfactory fit with a two component model power law $(\Gamma=1.51)$ plus Raymond-Smith thermal plasma $(k T=0.95 \mathrm{keV})$.

NGC 5846 The $\mathrm{X}$-ray morphology of this source is complex (see also Trinchieri \& Goudfrooij 2002), extending over more than $3^{\prime}$, almost to the limits of the optical galaxy. It exhibits two "spiral arms", directed to the Sortheast and Southwest. There is a weak hard X-ray nucleus, coincident with the VLA radio core in NGC 5846, whose spectrum was fit with a power law $(\Gamma=2.29)$. The source we have identified with the nucleus coincides with the third most luminous X-ray source in NGC 5846, listed as source number 12 in Trinchieri \& Goudfrooij (2002). Their quoted flux for this source is six times higher then our estimation because they convert counts to fluxes assuming a constant conversion factor. If we apply the same formulation, our results are consistent with theirs by a factor of 1.4. 
Table 3. X-ray properties (published or archival). Column 1: source name. Column 2: exposure time. Column 3: photon index (and Raymond-Smith plasma temperature) of the power law fit and assumed. Column 4: unabsorbed X-ray flux in the 0.5-2 keV band. Column 5: unabsorbed X-ray flux in the $2-10 \mathrm{keV}$ band. Column 6: logarithm of the soft X-ray luminosity (0.5-2 keV). Column 7: logarithm of the hard X-ray luminosity (2-10 keV). Column 8: note.

\begin{tabular}{|c|c|c|c|c|c|c|c|}
\hline $\begin{array}{l}\text { Galaxy } \\
\text { (1) }\end{array}$ & $\begin{array}{c}\tau \\
(\mathrm{ks}) \\
(2)\end{array}$ & $\begin{array}{c}\text { Model } \\
\Gamma(+k T) \\
(3) \\
\end{array}$ & $\begin{array}{c}F_{\text {soft }} \\
\left(\mathrm{erg} \mathrm{cm}^{-2} \mathrm{~s}^{-1}\right) \\
(4) \\
\end{array}$ & $\begin{array}{c}F_{\text {hard }} \\
\left(\mathrm{erg} \mathrm{cm}^{-2} \mathrm{~s}^{-1}\right) \\
(5)\end{array}$ & $\begin{array}{c}\log L_{\mathrm{soft}} \\
\left(\mathrm{erg} \mathrm{s}^{-1}\right) \\
(6) \\
\end{array}$ & $\begin{array}{c}\log L_{\text {hard }} \\
\left(\mathrm{erg} \mathrm{s}^{-1}\right) \\
(7) \\
\end{array}$ & $\begin{array}{c}\text { Note } \\
(8) \\
\end{array}$ \\
\hline N 660 & 1.92 & 1.7 & $7.12 \times 10^{-15}$ & $1.30 \times 10^{-14}$ & 38.07 & 38.33 & a \\
\hline N 1055 & 1.14 & 1.7 & $<7.11 \times 10^{-15}$ & $<1.30 \times 10^{-14}$ & $<38.13$ & $<38.39$ & $\mathrm{a}, \mathrm{b}$ \\
\hline N 3245 & 9.63 & 1.7 & $7.43 \times 10^{-15}$ & $1.36 \times 10^{-14}$ & 38.63 & 38.89 & $\mathrm{a}$ \\
\hline N 3627 & 1.75 & 1.7 & $\lesssim 1.20 \times 10^{-14}$ & $\lesssim 2.19 \times 10^{-14}$ & $\$ 37.79$ & $\lessgtr 38.06$ & $\mathrm{a}, \mathrm{b}$ \\
\hline N 4552 & 54.42 & $1.51(0.95)$ & $4.81 \times 10^{-14}$ & $7.58 \times 10^{-14}$ & 39.21 & 39.41 & $\mathrm{c}$ \\
\hline N 5846 & 29.86 & 2.29 & $3.35 \times 10^{-15}$ & $2.44 \times 10^{-15}$ & 38.51 & 38.37 & $\mathrm{~d}, \mathrm{e}$ \\
\hline N 5866 & 2.25 & 2.0 & $\ldots$ & $<6.37 \times 10^{-15}$ & $\ldots$ & $<38.26$ & $\mathrm{a}, \mathrm{f}$ \\
\hline N7331 & 29.46 & 1.46 & $4.46 \times 10^{-15}$ & $1.16 \times 10^{-14}$ & 38.04 & 38.45 & g \\
\hline
\end{tabular}

NOTES - (a) Photon index fixed to the respective value; (b) also in Ho et al. (2001); (c) error in the photon index is \pm 0.09 ; (d) also in Trinchieri $\&$ Goudfrooij (2002); (e) error in the photon index is \pm 0.38 ; (f) quoted Chandra ACIS-S data from Terashima \& Wilson (2003); (g) error in the photon index is \pm 0.32 .

NGC 5866 Recent published Chandra observations of this source failed to detect any hard X-ray emission from the nucleus (Terashima \& Wilson 2003).

NGC 7331 The Chandra soft X-ray image of this galaxy shows several blotchy regions of emission, along about $3^{\prime}$, in a North-South direction, similar to the morphology of the optical galaxy and also the radio (Cowan et al. 1994). Previous observations with ROSAT (Stockdale et al. 1998) have detected a luminous X-ray nucleus (few $10^{40} \mathrm{erg} \mathrm{s}^{-1}$ ). The Chandra observation has resolved the ROSAT emission into several X-ray sources and revealed a hard X-ray nucleus positionally coincident with the radio core detected in Cowan et al. (1994). The spectrum of the nuclear source is fit with a one component power law $(\Gamma=1.46)$ model and the luminosity is two magnitudes lower $\left(\sim 10^{38} \mathrm{erg} \mathrm{s}^{-1}\right)$ than the ROSAT value.

\section{Radio, optical and X-ray relations}

In Table 4 we summarize the relevant properties for the sample sources from published, archival or new observations.

With the exception of NGC 3245 (Barth et al. 2001), there are no reliable dynamical black hole mass estimates for the sample sources. The next best method for determining black hole mass utilizes the central velocity dispersion. The correlation between black hole mass and central velocity dispersion is tight (Ferrarese \& Merritt 2000; Gebhardt et al. 2000; Tremaine et al. 2002):

$\log \left(\frac{M_{\mathrm{BH}}}{M_{\odot}}\right)=8.13+4.02 \log \left[\frac{\sigma_{\mathrm{e}}}{200 \mathrm{kms}^{-1}}\right]$

where $\sigma_{\mathrm{e}}$ is the luminosity-weighted stellar velocity dispersion measured at the effective radius. However, in this case we will simply use the value $\sigma_{\mathrm{c}}$, the central velocity dispersion, since Gebhardt et al. (2000) have shown that the difference between $\sigma_{\mathrm{e}}$ and $\sigma_{\mathrm{c}}$ is only $\sim 10 \%$.

As can be seen in Table 4, the dynamical black hole mass estimate for NGC $3245\left(2 \times 10^{8} M_{\odot}\right.$; Barth et al. 2001) is in excellent agreement with the velocity dispersion estimate.

Nuclear $B$-band magnitudes have been calculated using the correlation found for low luminosity Seyfert 1 s between $M_{B}^{\text {nuc }}$ and the $\mathrm{H} \beta$ (narrow plus broad) line luminosity (Ho \& Peng 2001):

$\log \left(L_{\mathrm{H} \beta}\right)=-0.34 M_{B}^{\mathrm{nuc}}+35.1$.

With the exception of the Type 1.9 (broad $\mathrm{H} \alpha$ emission present) source NGC 1161, all the sample sources are Type 2 (no broad-line emission). Therefore, we have used only the narrow $\mathrm{H} \beta$ line luminosities taken from Ho et al. (1997a) to estimate $M_{B}^{\text {nuc }}$. In the case of composite galaxies, the derived $M_{B}^{\text {nuc }}$ should be taken as an upper limit, due to contamination of the nucleus by stellar light and obscuration of the $\mathrm{H} \beta$ line.

The radio-loudness parameter $R_{\mathrm{o}}$, was calculated as the ratio of the $5 \mathrm{GHz}$, mas-scale radio (except for NGC 7331) to nuclear $B$-band luminosity.

In comparison, the radio-loudness parameter $R_{\mathrm{x}}$ was calculated as the ratio of the $5 \mathrm{GHz}$, mas-scale radio (except for NGC 7331) to hard X-ray luminosity, as formulated in Terashima \& Wilson (2003).

The Eddington luminosity - the maximum luminosity output of an accreting object in an isotropic homogeneous system - was calculated as (in units of $\operatorname{erg~s}^{-1}$ ):

$L_{\mathrm{Edd}}=1.3 \times 10^{38}\left(\frac{M_{\mathrm{BH}}}{M_{\odot}}\right)$. 
Table 4. Summary of the AGN candidate properties (published, archival or new). Column 1: source name. Column 2: adopted distance for $H_{0}=75 \mathrm{~km} \mathrm{~s}^{-1} \mathrm{Mpc}^{-1}$ (Tully 1988 or Ho et al. $1997 \mathrm{a}$ ). Columns 3-5: extinction-corrected [OI] $\lambda 6300, \mathrm{H} \alpha$, and $\mathrm{H} \beta$ line luminosity (Ho et al. 1997a, 2003). Column 6: published or new high resolution VLBA 5 GHz (or VLA; see footnote) radio core luminosity. When multiple $5 \mathrm{GHz}$, VLBA observations are available, the longest integration time observations is quoted. Column 7: archival and/or published Chandra hard X-ray (2-10 keV) nuclear luminosity. Column 8: nuclear $B$-band magnitude. Column 9: logarithm of the radio to nuclear $B$ band ratio. Column 10: logarithm of the radio to hard X-ray ratio. Column 11: logarithm of the X-ray to $\mathrm{H} \alpha$ ratio. Column 12: central velocity dispersion (Lyon/Meudon Extragalactic Database or HYPERCAT - NGC 660, 3627, 4419, 4527, and 5354). Column 13: estimated black hole mass obtained from the velocity dispersion. Column 14: logarithm of the Eddington luminosity. Column 15: logarithm of the X-ray to Eddingtion luminosity ratio.

\begin{tabular}{|c|c|c|c|c|c|c|c|c|c|c|c|c|c|c|}
\hline $\begin{array}{l}\text { Galaxy } \\
\text { (1) }\end{array}$ & $\begin{array}{c}D \\
(\mathrm{Mpc}) \\
(2)\end{array}$ & $\begin{array}{c}\log L([\mathrm{O} \mathrm{I}]) \\
\left(\mathrm{erg} \mathrm{s}^{-1}\right) \\
(3)\end{array}$ & $\begin{array}{c}\log L(\mathrm{H} \alpha) \\
\left(\mathrm{erg} \mathrm{s}^{-1}\right) \\
(4)\end{array}$ & $\begin{array}{c}\log L(\mathrm{H} \beta) \\
\left(\mathrm{erg} \mathrm{s}^{-1}\right) \\
(5)\end{array}$ & $\begin{array}{c}\log L_{\mathrm{R}} \\
\left(\operatorname{erg~s}^{-1}\right) \\
(6)\end{array}$ & $\begin{array}{c}\log L_{X} \\
\left(\operatorname{erg~s}^{-1}\right) \\
(7)\end{array}$ & $\begin{array}{c}M_{B}^{\text {nuc }} \\
(\mathrm{mag}) \\
(8)\end{array}$ & $\begin{array}{l}\frac{L_{\mathrm{R}}}{L_{0}} \\
(9)\end{array}$ & $\begin{array}{c}\frac{L_{\mathrm{R}}}{L_{\mathrm{X}}} \\
(10)\end{array}$ & $\begin{array}{l}\frac{L_{\mathrm{X}}}{L(\mathrm{H} \alpha)} \\
(11)\end{array}$ & $\begin{array}{c}\sigma_{\mathrm{c}} \\
\left(\mathrm{km} \mathrm{s}^{-1}\right) \\
(12)\end{array}$ & $\begin{array}{c}M_{\mathrm{BH}} \\
\left(M_{\odot}\right) \\
(13)\end{array}$ & $\begin{array}{c}\log L_{\text {Edd }} \\
\left(\operatorname{erg~s}^{-1}\right) \\
(14)\end{array}$ & $\begin{array}{l}\frac{L_{\mathrm{X}}}{L_{\mathrm{Edd}}} \\
(15)\end{array}$ \\
\hline N410 & 70.6 & $<38.4$ & 39.43 & 39.46 & $<37.08$ & $\ldots$ & -12.82 & $<1.6$ & $\ldots$ & $<2.6$ & 285 & $5.5 \times 10^{8}$ & 46.85 & \\
\hline N 524 & 67.0 & $<37.7$ & 38.58 & 38.08 & 37.61 & & -8.76 & 3.7 & & & 245 & $3.1 \times 10^{8}$ & 46.60 & \\
\hline N 660 & 11.8 & 39.1 & 40.41 & 39.27 & $<35.62$ & 38.33 & -12.26 & $<0.3$ & $<-2.7$ & $<-2.1$ & 128 & $2.2 \times 10^{7}$ & 45.46 & -7.1 \\
\hline N 1055 & 12.6 & 36.7 & 37.92 & $\ldots$ & $<35.58$ & $<38.39$ & $\ldots$ & $\ldots$ & $\ldots$ & $<0.5$ & 80 & $3.4 \times 10^{6}$ & 44.65 & $<-6.3$ \\
\hline N 1161 & 25.9 & $<38.2$ & 39.01 & 38.39 & $<36.31$ & $\ldots$ & -9.68 & $<2.1$ & $\ldots$ & $<2.3$ & 285 & $5.6 \times 10^{8}$ & 46.86 & \\
\hline N 3245 & 22.2 & 39.0 & 40.03 & 39.35 & $<36.17$ & 38.89 & -12.50 & $<0.8$ & $<-2.7$ & $<-1.1$ & 211 & $1.7 \times 10^{8}$ & 46.34 & -7.5 \\
\hline N 3627 & 6.6 & 38.3 & 39.16 & 38.39 & $<34.90$ & $\lesssim 38.06$ & -9.68 & $<0.7$ & $\sim-3.2$ & $<-1.1$ & 124 & $2.0 \times 10^{7}$ & 45.44 & $\lesssim-7.4$ \\
\hline N 4216 & 16.8 & 38.0 & 38.89 & 38.25 & $<35.83$ & $\ldots$ & -9.26 & $<1.8$ & $\ldots$ & $\ldots$ & 219 & $1.9 \times 10^{8}$ & 46.39 & $\cdots$ \\
\hline N 4419 & 16.8 & 38.1 & 40.72 & 39.61 & $<35.83$ & $\ldots$ & -13.26 & $<0.2$ & $\ldots$ & & 101 & $8.7 \times 10^{6}$ & 45.05 & \\
\hline N 4527 & 13.5 & 39.0 & 40.12 & 39.12 & $<35.74$ & $\ldots$ & -11.82 & $<0.6$ & $\ldots$ & & 214 & $1.8 \times 10^{8}$ & 46.37 & \\
\hline $\mathrm{N} 4552^{a}$ & 16.8 & $<37.7$ & 38.55 & 38.05 & 38.23 & 39.41 & -8.68 & 4.4 & -1.2 & 0.9 & 264 & $4.1 \times 10^{8}$ & 46.73 & -7.3 \\
\hline N 5354 & 32.8 & 37.9 & 38.71 & 38.26 & 37.63 & $\ldots$ & -9.29 & 3.5 & $\ldots$ & $\ldots$ & 221 & $2.0 \times 10^{8}$ & 46.41 & $\ldots$ \\
\hline N 5838 & 28.5 & $>37.7$ & 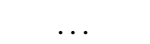 & $\ldots$ & $<36.17$ & $\ldots$ & $\ldots$ & $\ldots$ & $\ldots$ & $\ldots$ & 297 & $6.6 \times 10^{8}$ & 46.93 & $\ldots$ \\
\hline $\mathrm{N} 5846^{b, c}$ & 28.5 & 38.0 & 39.01 & 38.43 & 36.73 & 38.37 & -9.79 & 2.4 & -1.6 & -0.7 & 250 & $3.3 \times 10^{8}$ & 46.63 & -8.3 \\
\hline $\mathrm{N} 5866^{d}$ & 15.3 & $<37.9$ & 38.82 & 37.99 & 37.07 & $<38.26$ & -8.50 & 3.3 & $>-1.2$ & -0.6 & 142 & $3.4 \times 10^{7}$ & 45.65 & $<-7.4$ \\
\hline $\mathrm{N} 7331^{e}$ & 14.3 & $<37.7$ & 38.73 & 38.14 & $<35.17$ & 38.45 & -8.94 & $<1.2$ & $<-3.3$ & -0.3 & 146 & $3.8 \times 10^{7}$ & 45.69 & -7.2 \\
\hline
\end{tabular}

${ }^{a}$ VLBA, $5 \mathrm{GHz}, 2$ mas resolution radio core luminosity from Nagar et al. (2002).

${ }^{b}$ VLBA, $5 \mathrm{GHz}, 2$ mas resolution radio core luminosity given for the hypothetical core - component $\mathrm{A}$

${ }^{c}$ Hard X-ray (2-10 keV) luminosity from Terashima \& Wilson (2003).

${ }^{d}$ VLBA, $5 \mathrm{GHz}, 2$ mas resolution radio core luminosity from Falcke et al. (2002).

${ }^{e}$ VLA, $5 \mathrm{GHz}, 2 "$ resolution radio core luminosity from Cowan et al. (1994). 


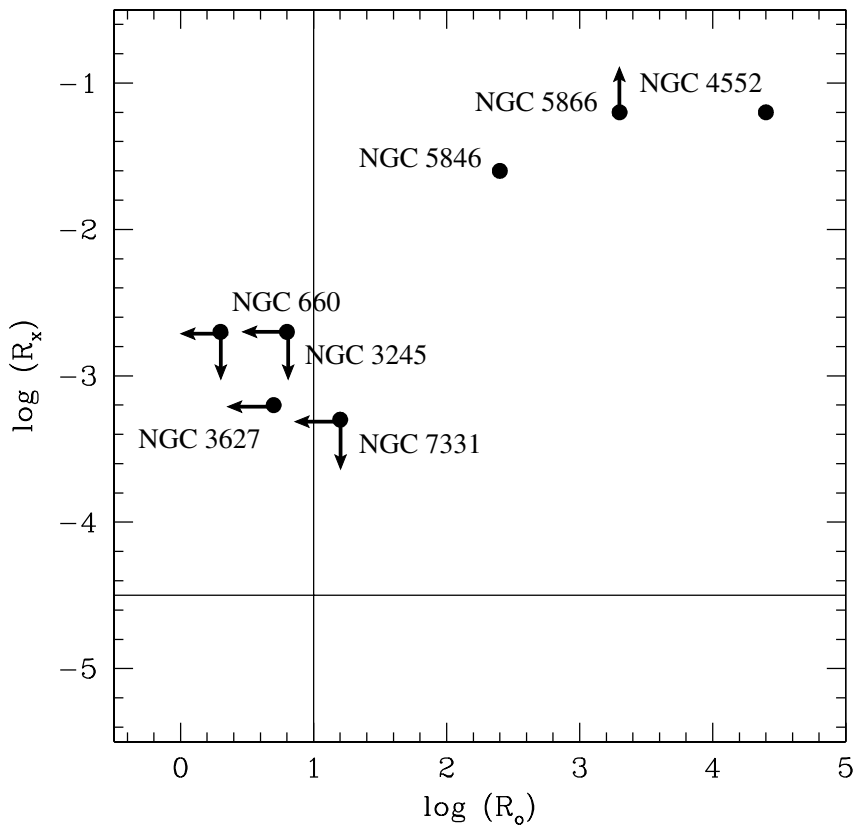

Fig. 3. The radio to X-ray ratio $\left(R_{\mathrm{x}}\right)$ versus radio to optical $\left(R_{\mathrm{o}}\right)$ relation in logarithmic units. Upper limits in X-ray and radio luminosity are illustrated by arrows. The solid lines define the radio-loud/radioquiet boundary for the optical and X-ray diagnostic.

\subsection{Radio-loudness}

Objects with $\log R_{\mathrm{O}}>1$ are commonly said to be radio-loud (e.g. Kellermann et al. 1994). Ho \& Peng (2001) have recently challenged the long-thought notion that Seyfert galaxies are mainly radio-quiet galaxies. By using nuclear $B$-band magnitudes and radio powers, they found that as many as $60 \%$ of Seyferts are radio-loud.

An alternative method for parameterizing radio-loudness involves using the radio to hard X-ray ratio (Terashima \& Wilson 2003). This method has the advantage of suffering less from extinction than the optical and allows the identification of the nucleus by means of high spatial resolution observations (e.g. Chandra). The equivalent radio-loud/radio-quiet boundary is established at $\log R_{\mathrm{x}}=-4.5$ (Terashima \& Wilson 2003).

In Fig. 3 we have plotted the logarithm of the $R_{\mathrm{x}}$ versus $R_{\mathrm{o}}$ parameters, where the solid lines denote the radioloud/radio-quiet boundaries.

NGC 660, NGC 3245, NGC 3627 and NGC 7331 are found to be optically radio-quiet. The remaining sources are radioloud, according to the $\log R_{\mathrm{o}}>1$ criterion (e.g. Kellermann et al. 1994). In particular, all of the mas-scale radio cores, as shown by the $5 \mathrm{GHz}$ VLBA observations, are radio-loud. Our results support those of Ho \& Peng (2001), whereby radio-loud objects are also found in disk-dominated hosts (see Filho et al. 2000, 2002).

As can be seen from the plot as well as Table 4, with the possible exception of NGC 660, NGC 3245 and NGC 7331, all sources are radio-loud according to the $\log R_{\mathrm{x}}>-4.5$ criterion. In particular, all the detected mas-scale cores are radio-loud.

The $R_{\mathrm{O}}$ and $R_{\mathrm{x}}$ results are consistent, in that all mas-scale radio cores are radio-loud. However, differences in the remaining sources most likely arise from stellar contribution. It is to be noted, for example, the high $\mathrm{H} \alpha$ luminosities associated with NGC 660, NGC 3245, and NGC 3627.

\subsection{Radio vs. $\mathrm{H} \alpha$ and [O I] luminosity}

It is well known that classical active galaxies like Seyferts, radio galaxies and LINERs display correlations between their radio and emission-line properties (Ho \& Ulvestad 2001; Ulvestad \& Ho 2001a; Zirbel \& Baum 1995; Nagar et al. 2000, 2002). In particular, Nagar et al. $(2000,2002)$ also show that there is a correlation between the radio core luminosity and [O I] $\lambda 6300$ line luminosity in LINERs and low luminosity Seyferts.

The radio luminosity of the present sample does not seem to correlate with $\mathrm{H} \alpha$ or with [O I] $\lambda 6300$ luminosity, but because of the small sample and large intrinsic scatter in the correlation we are not able to draw any conclusions. The absence of a radio and emission-line correlation for composite sources had already been noted in Filho et al. (2002) using lower resolution, VLA radio observations.

\subsection{X-Ray vs. Ho luminosity}

By examining the hard $\mathrm{X}$-ray to $\mathrm{H} \alpha$ luminosity ratio, we wish to investigate whether the observed X-ray emission is related to the continuum source which powers the emission lines.

Such a correlation between the X-ray and $\mathrm{H} \alpha$ luminosities is observed in classical AGN such as Seyferts and quasars (Ward et al. 1988) and more recently in LINER 1s (Terashima et al. 2000; Ho et al. 2001), indicating that the AGN is capable of powering the optical emission lines. Typical ratios for LLAGN are in the range:

$\log \left(\frac{L_{\mathrm{X}}}{L_{\mathrm{H} \alpha}}\right) \sim 1-2$.

In Fig. 4 we plot the relation for our sample sources, where the X-ray luminosity is taken from the hard X-ray band. The shaded area defines ratios that are typical for AGN-powered sources.

All of the sources fall short of the empirical X-ray to $\mathrm{H} \alpha$ ratio observed in classical AGN, indicating that the observed $\mathrm{X}$-ray luminosities are too low to generate the $\mathrm{H} \alpha$ lines. There are three possible scenarios - either the emission lines are powered by a source other than the AGN, the ionizing spectrum is different than that inferred, or the X-ray emission is heavily obscured at energies above $2 \mathrm{keV}$. The first possibility has already been investigated for example in the work of Maoz et al. (1998), whereby circumnuclear stellar populations may provide enough ionizing photons to explain the observed optical emission-line flux. However, as investigated by Ho et al. (2003; see also Ho et al. 2001), there is no evidence for the presence of young stellar clusters. Alternatively, it may be that the underlying ionizing spectrum is more complicated then assumed - most of the ionizing photons may occur in the extreme ultraviolet (EUV) and soft X-ray region, not in the hard $\mathrm{X}$-rays. Powering of the emission lines from a central AGN could still occur if the hard X-ray flux were being heavily absorbed (Terashima et al. 2000; Terashima \& Wilson 2003). 


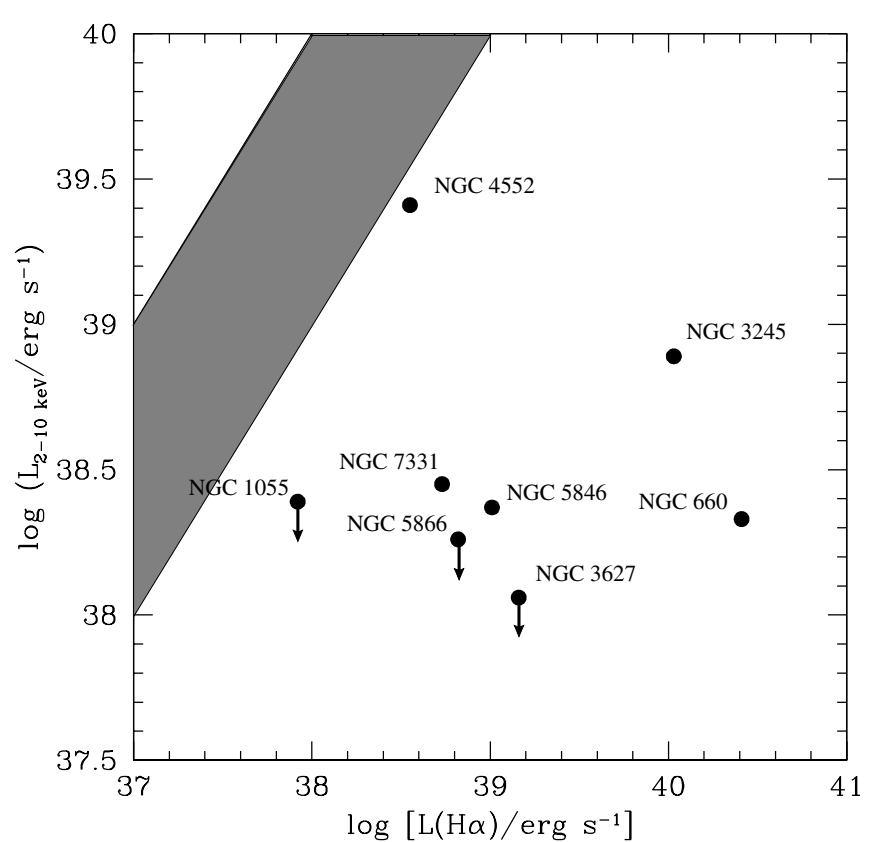

Fig. 4. The relation between hard X-ray core and $\mathrm{H} \alpha$ emission line luminosity. Upper limits in X-ray luminosity are illustrated by arrows. The shaded area corresponds to typical values for LLAGN $\log \left(\frac{L_{\mathrm{X}}}{L_{\mathrm{H} \alpha}}\right) \sim 1-2$.

If intrinsic X-ray luminosities are 1-2 orders of magnitude higher, then the X-ray to $\mathrm{H} \alpha$ ratio would be typical for LLAGN (Terashima \& Wilson 2003). Although scattering is still a viable explanation, observations at energies above $10 \mathrm{keV}$ are needed in order to observe any Compton-thick component.

\subsection{Radio vs. X-ray luminosity}

In Fig. 5 we plot the mas-scale radio core luminosity versus the hard X-ray core luminosity for the sample sources.

It can be seen from the plot that the detected mas-scale radio cores have $L_{\mathrm{R}}>10^{36.5} \mathrm{erg} \mathrm{s}^{-1}$, with two sources (NGC 4552 and NGC 5846) being firm Chandra detections (Table 3). Three of the sources with no mas-scale radio detection, however, show hard X-ray Chandra detections NGC 660, NGC 3245 and NGC 7331. The Chandra observations of NGC 1055 and NGC 3627 (see also Ho et al. 2001) failed to detect any hard X-ray nuclear source, most likely due to the low integration time for the X-ray observations $(\tau<2 \mathrm{ks}$; Table 3). Combining both the radio and X-ray data yields a $\sim 50 \%$ LLAGN detection rate among the sample sources or equivalently a $12 \%$ detection rate for the entire composite LINER/H II galaxy sample.

NGC 7331 has a very low luminosity VLA radio core (Cowan et al. 1994), 10 times that of Sgr A*, and also the lowest hard X-ray core luminosity of all the sample sources, $10^{4}$ times that of $\mathrm{Sgr} \mathrm{A}^{*}$. Similarities in the arcsec-scale radio properties - radio morphology and low radio flux density - of NGC 7331 and the cores that were not detected on mas-scales, indicate that NGC 7331 may actually be a prototype. These

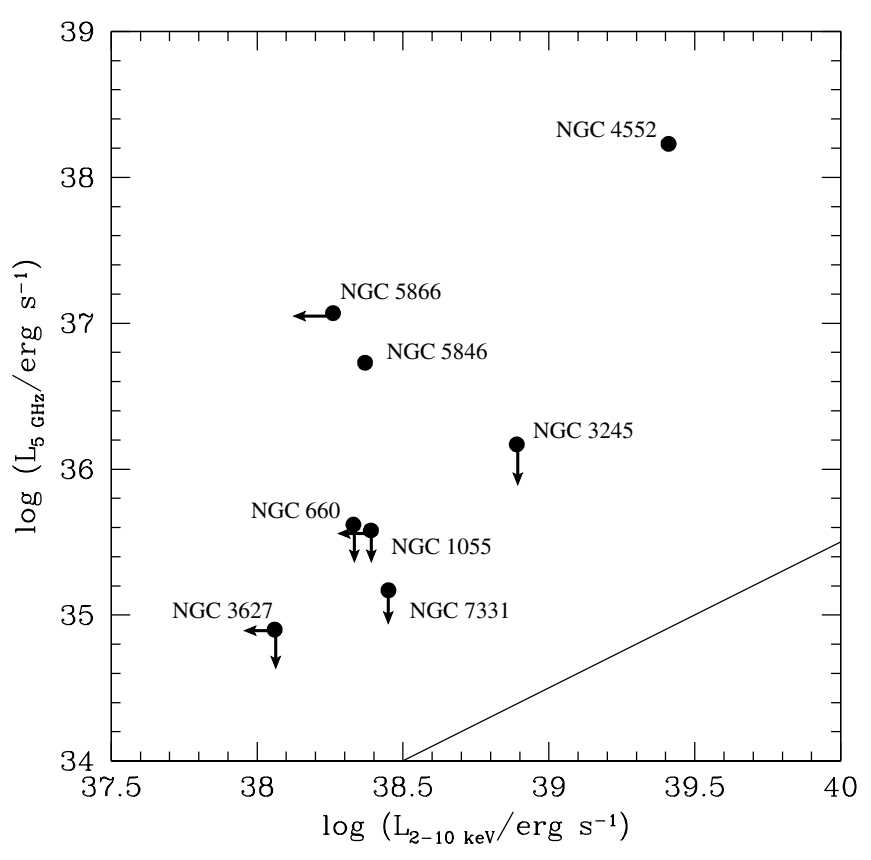

Fig. 5. The relation between the mas-scale radio core and hard X-ray core luminosity. Upper limits in radio and X-ray luminosity are illustrated by arrows. The solid line defines the radio-loud/radio-quiet boundary for the X-ray diagnostic.

sources may all harbour submillijansky radio and low luminosity hard X-ray AGN cores, difficult to detect unless deep observations are obtained. Nevertheless, the results demonstrate the combined power of high resolution X-ray and radio imaging in LLAGN detection. We will return to this issue in the next section.

\section{ADAFs or jets?}

We wish to use our observations to test models of radio and $\mathrm{X}$-ray emission in low luminosity sources, namely advectiondominated accretion flows (ADAFs) and jet models. These models have been extensively applied to several other low luminosity sources, of which Sgr A* (Yuan et al. 2002a, 2003) and NGC 4258 (Yuan et al. 2002b) are just two examples.

ADAFs (see reviews by Narayan et al. 1998 and Quataert 2001) occur in the low accretion rate regime $\left(m_{\text {acc }}\right.$ (crit) $<10^{-1.6}$, where $m_{\text {acc }}$ is given in dimensionless units of Eddington accretion rate). They are characterized by a low radiative efficiency, due to the advection of a significant amount of the energy into the black hole. ADAF theory has developed quickly in recent years with the inclusion of outflows and the possible existence of nonthermal electrons (see Yuan et al. 2003 for a review). However, the analysis and therefore conclusions of this paper are based on the canonical ADAF model.

ADAFs may be able to account for correlations observed between the radio and X-ray emission. Particularly, this type of low radiative efficiency flow is supported by the highly subEddington luminosities observed in our sources $\left(\frac{L_{\mathrm{X}}}{L_{\mathrm{Fdd}}}<10^{-5}\right.$; Table 4). Furthermore, ADAFs are naturally bright in radio 


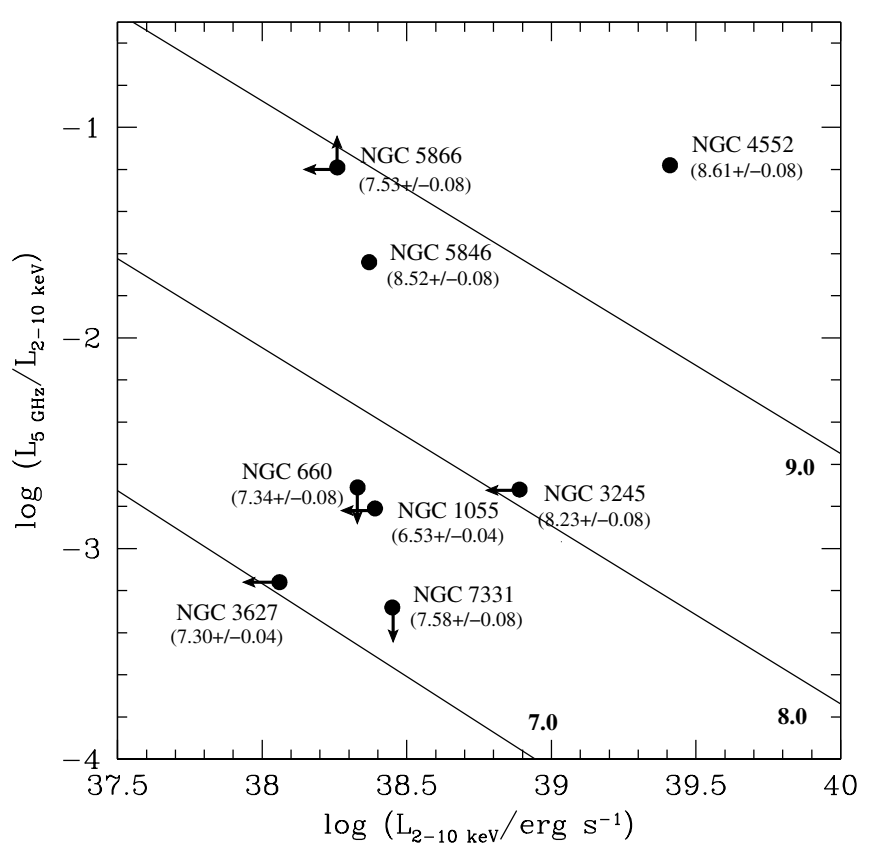

Fig. 6. The relation between the mas-scale radio core/hard X-ray core luminosity ratio $\left(L_{5 \mathrm{GHz}} / L_{2-10 \mathrm{keV}}\right)$ and the hard X-ray core luminosity. Upper limits in radio and X-ray luminosity are illustrated by arrows. Numbers in parentheses refer to the logarithm of the black hole masses and the respective error, considering an error in $\log \sigma_{\mathrm{c}}=0.06,0.02$ and 0.01 plus a $10 \%$ error in the conversion of $\sigma_{\mathrm{e}}$ to $\sigma_{\mathrm{c}}$. The solid lines refer to the ADAF model prediction $-L_{\mathrm{R}} \propto M_{\mathrm{BH}} L_{\mathrm{X}}^{0.1}-$ for several black hole masses $-\log M_{\mathrm{BH}}=7.0,8.0,9.0-$ in logarithmic units of solar mass.

due to radio synchrotron cooling and dim in the UV/optical regime due to the absence of a "blue bump", the signature of a standard, optically thick but geometrically thin accretion disk (Shakura \& Sunyaev 1973). Both of these ADAF characteristics conspire to produce "radio-loud" cores.

In ADAFs the hard X-ray emission arises from bremsstrahlung and Compton scattering of synchrotron photons subject to self-absorption. As a result, the ADAF model predicts the correlation (Yi \& Boughn 1998, 1999):

$L_{\mathrm{R}} \propto M_{\mathrm{BH}} L_{\mathrm{X}}^{0.1}$.

In Fig. 6 we plot the mas-scale radio core to hard X-ray ratio versus the hard X-ray core luminosity. In parentheses are the logarithms of the black hole mass plus error estimations, which include the error in velocity dispersion plus a $10 \%$ error associated with the conversion from $\sigma_{\mathrm{e}}$ to $\sigma_{\mathrm{c}}$. The lines refer to the ADAF model prediction for different black hole masses, appropriately scaled to $5 \mathrm{GHz}$.

If the sources with upper limits to their mas-scale radio emission contain accreting black holes as is the case for NGC 7331 (due to a hard X-ray detection), then it is possible to explain their radio emission using the ADAF model, as originating in underfed black holes $\left(m_{\mathrm{acc}}<10^{-3}\right)$. However, for the mas-scale detected radio cores, the ADAF model overpredicts the black hole masses by a factor 10 or so (Table 4) even when the errors associated with the black hole mass determination are considered (Fig. 6). This suggests that the ADAF model is

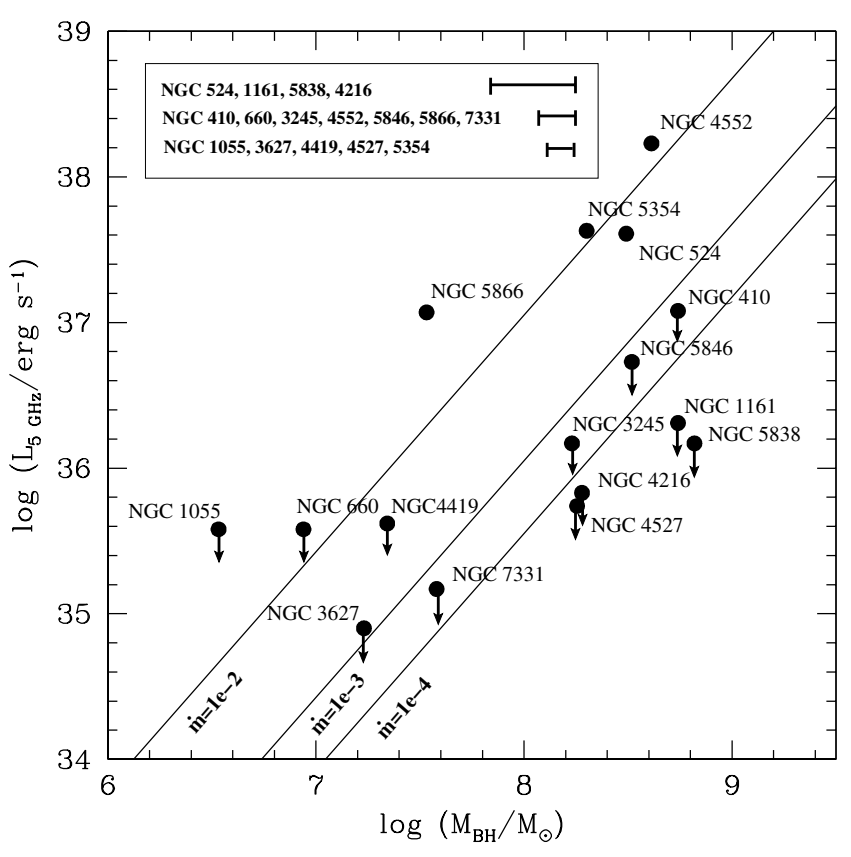

Fig. 7. The relation between the mas-scale radio core luminosity and the black hole mass. Upper limits in radio luminosity are illustrated by arrows. We give typical error bars for the black hole mass, considering an error in $\log \sigma_{\mathrm{c}}=0.06,0.02$ and 0.01 plus a $10 \%$ error in the conversion of $\sigma_{\mathrm{e}}$ to $\sigma_{\mathrm{c}}$. The solid lines refer to the ADAF model prediction $-L_{\mathrm{R}} \propto m_{\mathrm{acc}}^{1.2} M_{\mathrm{BH}}^{1.6}$ - for different mass accretion rates $m_{\text {acc }}=10^{-2}, 10^{-3}, 10^{-4}-$ given in dimensionless units of Eddington accretion rate.

not entirely consistent with the observed radio to X-ray ratio unless the hard X-rays are heavily obscured (see Sect. 5.3).

In Fig. 7 we plot the mas-scale radio core luminosity versus the black hole mass. The solid lines refer to the ADAF prediction of:

$L_{\mathrm{R}} \propto m_{\mathrm{acc}}^{1.2} M_{\mathrm{BH}}^{1.6}$

where the relation has been appropriately scaled to $5 \mathrm{GHz}$. Typical errors in black hole mass are given.

The results show that composite sources with masscale radio cores have a radio luminosity to black hole mass ratio similar to low luminosity Seyferts and LINERs $\left(\log \left(L_{\mathrm{R}} / M_{\mathrm{BH}}\right) \sim 1.14\right.$; Nagar et al. 2002). On the other hand, if the sources with no mas-scale radio detection harbour an $\mathrm{AGN}$, then on average they should have lower radio to black hole ratios than the LLAGN in the Nagar et al. (2002) sample.

Figure 7 also shows the ADAF model prediction for several accretion rates. Sources with upper limits to their masscale radio emission are consistent with low mass accretion rates $\left(m_{\mathrm{acc}}<10^{-2}\right)$. However, the detected mas-scale cores fall very close to the ADAF permitted accretion limit of $m_{\text {acc }}$ (crit) $=10^{-1.6}$. These results suggest that the mas-scale radio cores show more radio emission than can perhaps be accounted for from an ADAF.

Plotted in Fig. 8 is the relation between the hard X-ray luminosity and the black hole mass. The solid lines refer to the 


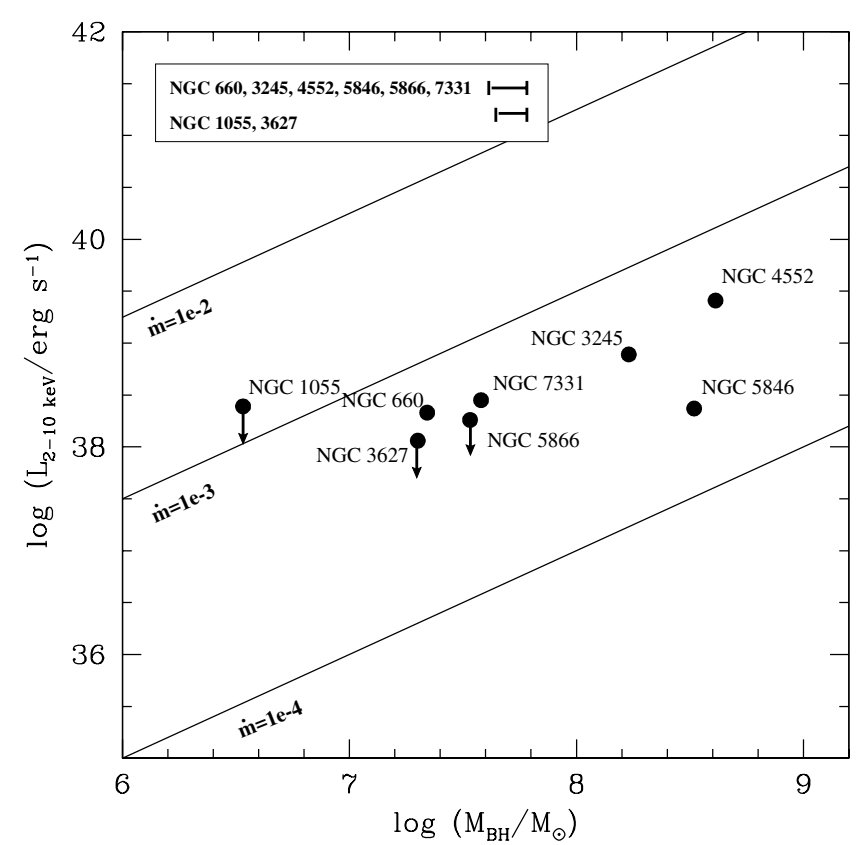

Fig. 8. The relation between the hard X-ray core luminosity and black hole mass. Upper limits in X-ray luminosity are illustrated by arrows. We give typical error bars for the black hole mass, considering an error in $\log \sigma_{\mathrm{c}}=0.06,0.02$ and 0.01 plus a $10 \%$ error in the conversion of $\sigma_{\mathrm{e}}$ to $\sigma_{\mathrm{c}}$. The solid lines refer to the ADAF model prediction $L_{\mathrm{X}} \propto M_{\mathrm{BH}} m_{\mathrm{acc}}^{2}$ - for various accretion rates $-m_{\mathrm{acc}}=10^{-2}, 10^{-3}, 10^{-4}$ given in dimensionless units of Eddington accretion rate.

predicted correlation in an ADAF model for various mass accretion rates:

$L_{\mathrm{X}} \propto M_{\mathrm{BH}} m_{\mathrm{acc}}^{\mathrm{x}}$

where $x=2$ for thermal emission. Again, typical errors in black hole mass determination are given in the plot.

The X-ray data are consistent with the ADAF model for low accretion rates $\left(m_{\mathrm{acc}}<10^{-3}\right)$, both for the sources with mas-scale upper limits and for those with mas-scale cores. This could suggest the same underlying mechanism for X-ray production in all sample sources - an ADAF. In fact, in two of the mas-scale radio cores where an X-ray spectral fit was possible (NGC 4552 and NGC 7331; see Table 3), the X-ray photon index is consistent with the predicted ADAF value of $\sim 1.4$.

In summary, if we take NGC 7331 as a prototypical submillijansky AGN core, then scaling arguments suggest that an ADAF could simultaneously explain both the radio and X-ray emission in the sources with no mas-scale radio detection. In fact, these sources may in fact be "bare" ADAFs, where the radio and X-ray contribution is coming mainly from the accretion flow. The absence of a jet or of an energetically significant contribution of a jet to the radio emission is not, however, clear. It may be that, like in M 81, the jet is present but very compact and difficult to detect (Bietenholz et al. 2000).

On the other hand, thermal ADAF models predict inverted radio spectra ( $\alpha \sim-0.4$; Narayan et al. 1998; see also Yuan et al. 2003) while the mas-scale sources possess flat spectrum radio cores (Table 2). Modified ADAF models that include convection (Ball et al. 2001) or outflows (Quataert \& Narayan 1999) do not provide satisfactory solutions, since the predicted spectral slopes are roughly the same as in the canonical ADAF models (Quataert \& Narayan 1999) ${ }^{1}$. Therefore, while the X-ray emission in mas-scale radio cores may be ADAF-powered, the radio emission may require another emission mechanism.

The most likely scenario is that the radio emission in the mas-scale detected radio cores is a result of an ADAF-type accretion (or variants thereof) plus a compact jet or outflow (Quataert et al. 1999; Yuan 2000; Falcke et al. 2001; Nagar et al. 2001; Ulvestad \& Ho 2001b; Nagar et al. 2002). The consideration of a jet is not ad hoc since we detect extended emission in 4 out of the 5 mas-scale radio cores. Furthermore, the addition of a self-absorbed jet component to an ADAF core may "flatten" out the spectral index to the observed values. In fact, flat spectrum radio emission in "classical" AGN are commonly attributed to the presence of jets.

In a recent paper, Falcke et al. (2004; see also Merloni et al. 2003) investigated an accretion rate-dependent scheme for black hole-powered sources, whereby sub-Eddington systems are predicted to be dominated by emission from a relativistic jet. Such an idea was suggested already in Falcke \& Biermann (1995), and a jet model was shown to analytically predict the radio/X-ray correlation seen in the low/hard state of the black hole candidate X-ray binary (XRB) GX 339-4 (Markoff et al. 2003). This correlation has now been seen in many other low/hard state XRBs, and is likely universal (Gallo et al. 2003). Falcke et al. (2004; see also Merloni et al. 2003) show that this correlation can be scaled with black hole mass to extend to certain classes of AGN as well. This leads to a radio/X-ray/black hole mass fundamental plane which unifies diverse sources such as FR I radio galaxies, LINERs and XRBs as jet-dominated (see Fig. 1 in Falcke et al. 2004).

This scaling relation is given by (Eq. (7); Falcke et al. 2004):

$L_{\mathrm{X}}^{\text {cor }} \propto L_{\mathrm{X}^{\prime}}\left(\frac{v_{\mathrm{X}^{\prime}}}{v_{\mathrm{X}}}\right)^{-\alpha_{\mathrm{X}}} M_{\mathrm{BH}}^{-m \alpha_{\mathrm{R}}+\alpha_{\mathrm{X}}}$

where $\alpha_{\mathrm{R}}$ and $\alpha_{\mathrm{X}}$ are the radio and X-ray spectral indices and $m$ is a function of both $\alpha_{\mathrm{R}}$ and $\alpha_{\mathrm{X}}$. We here apply the black hole mass scaling relation in order to investigate the presence of an energetically dominant jet in our sample sources. We have extrapolated our 2-10 keV luminosities (Table 3) to 3-9 keV assuming an average $\alpha_{\mathrm{X}}=0.6(\equiv \Gamma-1)$ and $\alpha_{\mathrm{R}}=-0.15$ (Falcke et al. 2004). The assumption of a spectral index of $\Gamma=1.6$ $\left(\alpha_{\mathrm{X}}=0.6\right)$ is consistent within $10 \%$ of values obtained using our actual tabulated spectral indices (Table 3 ) and therefore does not alter the general result.

In Fig. 9 we have plotted the scaled correlation normalized to the $6 M_{\odot}$ black hole mass of GX339-4. Typical errors in the "black hole mass-corrected" X-ray luminosity are conservatively estimated to be of the order of 0.08 in logarithmic units of luminosity.

1 The inclusion of power-law electrons has been recently proven to produce a flatter radio spectra (see Yuan et al. 2003). 


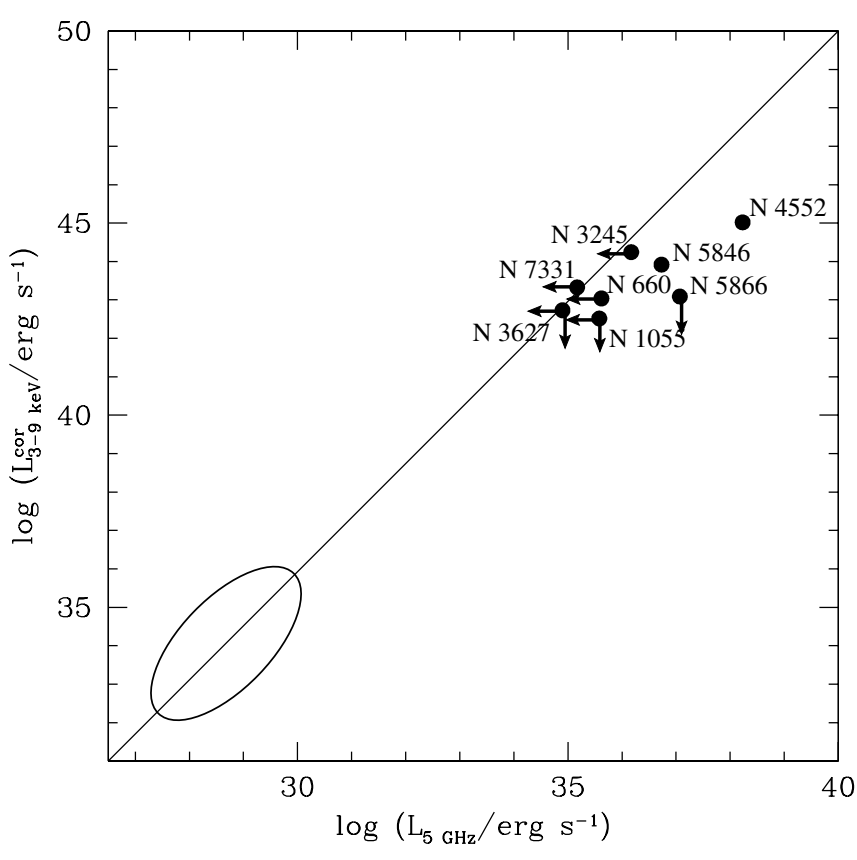

Fig. 9. The relation between radio and "black hole mass-corrected" X-ray luminosity, scaled to the $6 M_{\odot}$ black hole in XRB GX 339-4. The solid line is the predicted radio/X-ray correlation from the jet model and the ellipse details the broadband observations of GX 339-4 (Corbel et al. 2003; Markoff et al. 2003).

In addition to the scenario discussed above, a jet model alone seems to interpret the present data as well. It is noteworthy that the mas-scale detected sources fall very close to the jet model predicted relation. The deviation of the sources NGC 4552, NGC 5846 and NGC 5866 is in the direction of excess radio emission, which is entirely consistent with the jet model. The scaling relation is valid for the compact inner jet core while these sources are known to possess jet emission on mas-scales; hence, the radio flux density likely includes some extended jet emission.

\section{Conclusions}

We have embarked on a high resolution X-ray and radio imaging study of a sample of composite LINER/H II galaxies known to exhibit AGN-like properties. Five of the 16 sample sources possess milliarcsecond radio cores, four of which also show extended radio emission. Two of the sources with no milliarcsecond radio detection were found to possess hard X-ray nuclear cores. The $50 \%$ detection rate of radio and/or hard X-ray nuclei support the view that these AGN candidate composite $\mathrm{H}$ II galaxies host weak AGN central engines. When considering the entire composite LINER/H II sample, these results yield an overall $12 \%$ detection rate. Modeling of the radio and X-ray emission suggests that the mas-scale radio detected sources exhibit an energetically important contribution from a jet, associated with a sub-Eddingtion accreting black hole. The sources that were not detected on mas-scales, however, appear consistent with the radio and X-ray emission coming mainly from the low radiative efficiency accretion flow (ADAF). Nonetheless, even in the cases where the presence of an AGN is irrefutable, this AGN cannot be responsible solely for powering the observed emission lines unless the X-ray flux is heavily obscured.

Acknowledgements. M.E.F. acknowledges support from the Fundação para a Ciência e Tecnologia, Ministério da Ciência e Ensino Superior, Portugal through the grant PRAXIS XXI/BD/15830/98 and SFRH/BDP/11627/2002. We would like to thank Heino Falcke for the extensive discussions on ADAF and jet models and Jim Ulvestad for providing us with calibrated VLA data for NGC 660. We wish also to thank the anonymous referee for his helpful comments.

This research has made extensive use of NED (NASA/IPAC Extragalactic Database), which is operated by the Jet Propulsion Laboratory, California Institute of Tecnology, under contract with the National Aeronautics and Space Administration, LEDA (Lyon/Meudon Extragalactic Database), HYPERCAT and the Chandra data archive. The VLA and the VLBA are facilities of the National Radio Astronomy Observatory (NRAO) which is a facility of the National Science Foundation operated under cooperative agreement by Associated Universities, Inc.

\section{References}

Ball, G. H., Narayan, R., \& Quataert, E. 2001, ApJ, 552, 221

Barth, A. J., Sarzi, M., Rix, H.-W., et al. 2001, ApJ, 555, 685

Bietenholz, M. F., Bartel, N., \& Rupen, M. P. 2000, ApJ, 532, 895

Condon, J. J. 1992, ARA\&A, 30, 575

Corbel, S., Novak, M., Fender, R. P., Tzioumis, A. K., \& Markoff, S. 2003, A\&A, 400, 1007

Cowan, J. J., Romanishin, W., \& Branch, D. 1994, ApJ, 436, L139

Dickey, J. M., \& Lockman, F. J. 1990, ARA\&A, 28, 215

Falcke, H., \& Biermann, P. L. 1995, A\&A, 293, 665

Falcke, H., Nagar, N. M., Wilson, A. S., \& Ulvestad, J. 2000, ApJ, 542,197

Falcke, H., Nagar, N. M., Wilson, A. S., Ho, L. C., \& Ulvestad, J. S. 2001, in Black Holes in Binaries and Galactic Nuclei, ed. L. Kaper, E. P. J. van den Heuvel, \& P. A. Woudt (Springer), 218

Falcke, H., Körding, E., \& Markoff, S. 2004, A\&A, 414, 895

Ferrarese, L., \& Merritt, D. 2000, ApJ, 539, L9

Filho, M. E., Barthel, P. D., \& Ho, L. C. 2000, ApJS, 129, 93

Filho, M. E., Barthel, P. D., \& Ho, L. C. 2002, A\&A, 385, 425

Gebhardt, K., Bender, R., Bower, G., et al. 2000, ApJ, 539, L13

Ho, L. C. 1996, in The Physics of LINERs in View of Recent Observations, ed. M. Eracleous, A. Koratkar, C. Leitherer, \& L. Ho (San Francisco: ASP), 103

Ho, L. C., \& Peng, C. Y. 2001, ApJ, 555, 650

Ho, L. C., \& Ulvestad, J. S. 2001, ApJS, 133, 77

Ho, L. C., Filippenko, A. V., \& Sargent, W. L. W. 1997, ApJS, 112, 315

Ho, L. C., Feigelson, E. D., Townsley, L. K., et al. 2001, ApJ, 549, L51

Ho, L. C., Filippenko, A. V., \& Sargent, W. L. W. 2003, ApJ, 583, 159

Kellerman, K. I., Sramek, R. A., Schmidt, M., Green, R. F., \& Shaffer, D. B. 1994, AJ, 108, 1163

Maoz, D., Koratkar, A., Shields, J. C., et al. 1998, AJ, 116, 55

Markoff, S., Nowak, M., Corbel, S., Fender, R., \& Falcke, H. 2003, A\&A, 397, 645

Merloni, A., Di Matteo, T., \& Heinz, S. 2003

[arXiv:astro-ph/0305261] 
Nagar, N. M., Falcke, H., Wilson, A. S., \& Ho, L. C. 2000, ApJ, 542, 186

Nagar, N. M., Wilson, A. S., \& Falcke, H. 2001, ApJ, 559, 87

Nagar, N. M., Falcke, H., Wilson, A. S., \& Ulvestad, J. S. 2002, A\&A, 392,53

Narayan, R., Mahadevan, R., \& Quataert, E. 1998, in The Theory of Black Hole Accretion Discs, ed. M. A. Abramowicz, G. Björnsson, \& J. E. Pringle (Cambridge: Cambridge Univ. Press), 148

Perley, R. A., Schwab, F. R., \& Bridle, A. H. 1989, in Synthesis Imaging in Radio Astronomy: A Collection of Lectures from the Third NRAO Synthesis Imaging Summer School (San Francisco: ASP), 528

Quataert, E. 2001, in Probing the Physics of Active Galactic Nuclei by Multiwavelength Monitoring, ed. B. M. Peterson, R. S. Polidan, \& R. W. Pogge (San Francisco: ASP), 71

Quataert, E., \& Narayan, R. 1999, ApJ, 520, 298

Quataert, E., Di Matteo, T., Narayan, R., \& Ho, L. C. 1999, ApJ, 525, L89

Raymond, J. C., \& Smith, B. W. 1977, ApJS, 35, 419

Shakura, N. I., \& Sunyaev, R. A. 1973, A\&A, 24, 337
Stockdale, C. J., Romanishin, W., \& Cowan, J. J. 1998, ApJ, 508, L33

Tarchi, A., Neininger, N., Greve, A., et al. 2000, A\&A, 358, 95

Terashima, Y., Ho, L. C., \& Ptak, A. F. 2000, ApJ, 539, 161

Terashima, Y., \& Wilson, A. S. 2003, ApJ, 583, 145

Tremaine, S., Gebhardt, K., Bender, R., et al. 2002, ApJ, 574, 740

Trinchieri, G., \& Goodfrooij, P. 2002, A\&A, 386, 472

Ulvestad, J. S., \& Ho, L. C. 2001a, ApJ, 558, 561

Ulvestad, J. S., \& Ho, L. C. 2001b, ApJ, 562, L133

Ward, M. J., Done, C., Fabian, A. C., Tennant, A. F., \& Shafer, R. A. 1988, ApJ, 324, 767

Weiler, K. W., van der Hulst, J. M., Sramek, R. A., \& Panagia, N. 1981, ApJ, 243, L151

Yi, I., \& Boughn, S. P. 1998, ApJ, 499, 198

Yi, I., \& Boughn, S. P. 1999, ApJ, 515, 576

Yuan, F. 2000, MNRAS, 319, 1178

Yuan, F., Markoff, S., \& Falcke, H. 2002a, A\&A, 383, 854

Yuan, F., Markoff, S., \& Falcke, H., \& Biermann, P. L. 2002b, A\&A, 391, 139

Yuan, F., Quataert, E., \& Narayan, R. 2003 [arXiv:astro-ph/0304125]

Zirbel, E. L., \& Baum, S. A. 1995, ApJ, 448, 521 